\title{
CONTRIBUTION A L'ÉTUDE DES EFFETS BIOLOGIQUES DU MERCURE ET DE L'ARGENT DANS L'EAU DE MER
}

\author{
par J. SOYER
}

SOMMAIRE

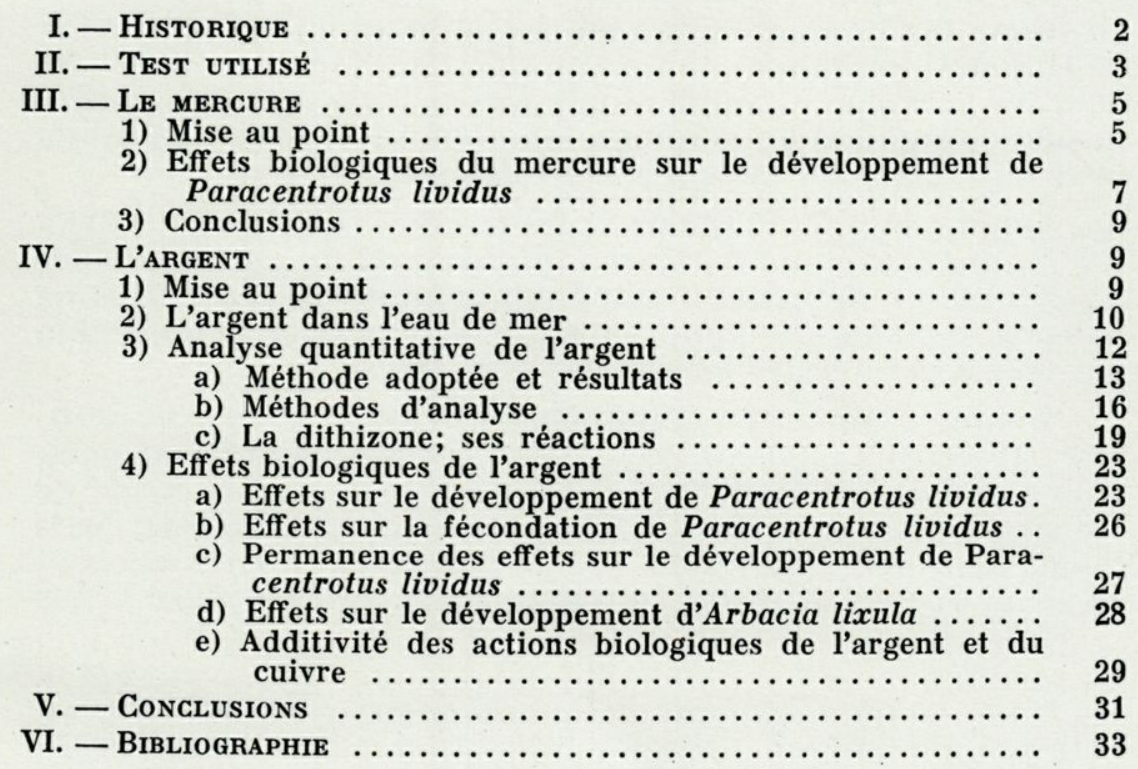




\section{HISTORIQUE}

La toxicité des sels de mercure et d'argent, connue depuis l'antiquité, a été mise à contribution dans les domaines de l'agriculture et de la médecine; aussi les publications qui s'y rapportent sont-elles innombrables. Par contre, les travaux concernant l'action biologique de ces deux métaux dans le domaine marin sont relativement rares. De plus, pendant longtemps, le matériel marin n'a été utilisé par les auteurs que pour mettre en évidence les propriétés toxiques de leurs sels, pour en déterminer les causes et pour trouver leur mode d'action. RicheT (1881), Lillie (1921), HoAdley (1923-1930), étudient, le premier sur des Poissons, les deux autres sur un Echinide, Arbacia aequituberculata, les effets du chlorure mercurique. DrzewINA et BoHN (1926-1931) fournissent un grand nombre de données concernant l'action nocive que l'argent exerce sur les plutéus d'Echinides et sur une Planaire du genre Convoluta.

Ce n'est qu'après les travaux de GaLtsoff (1932), qui mettent en évidence la concentration de certains ions métalliques par les organismes marins, que les chercheurs commencent à envisager le rôle possible que pouvaient jouer les métaux lourds dans le développement de ces organismes. Waterman (1937) est l'un des premiers à penser à une action biologique de ces éléments, notamment au voisinage des côtes où leur concentration peut augmenter de façon considérable.

Les travaux de Pyefinch et Mott (1944-1948) et de Clarke (1947) sur la toxicité des métaux utilisés dans les peintures dites " antifouling 》 destinées à empêcher la salissure des coques de bateaux, donnent des indications intéressantes sur la sensibilité des divers organismes fixés et de leurs larves.

Barnes et Stanbury (1948), Hunter (1949), Corner et SparRow (1956-1957) étudient l'action des éléments lourds sur quelques Crustacés et mettent en évidence l'influence du mode de vie sur la résistance des différentes espèces. Ces auteurs constatent également que les actions de ces métaux lourds peuvent s'ajouter et parfois même se renforcer, notamment dans le cas

* Nous tenons à remercier très sincèrement pour l'appui constant qu'il nous a prodigué, M. P. Bougrs, Professeur à la Faculté des Sciences de Paris, Sous-directeur de la Station Zoologique de Villefranche-sur-Mer où ce travail a été réalisé. 
de mélange cuivre-mercure, comme l'avaient déjà indiqué PyEFINCH et MotT.

Le mercure et l'argent, qui entrent dans la composition d'algues et d'animaux marins et qui sont toxiques à un seuil peu élevé, doivent intervenir en écologie, bien qu'ils soient présents dans l'eau de mer à des concentrations plus faibles que celles utilisées par les auteurs précédents. Il était vraisemblable de penser qu'il était possible, à l'aide de tests plus fins, d'observer l'action nocive de quantités de métal se rapprochant davantage des teneurs naturelles de l'eau de mer. Aussi nous a-t-il paru intéressant d'étudier l'action du mercure et de l'argent sur le développement de Paracentrotus lividus et d'Arbacia lixula, en essayant de déterminer avec précision leur seuil de toxicité afin d'avoir une idée du rôle que peuvent jouer ces deux éléments en écologie marine.

\section{TEST UTILISÉ}

Nous avons utilisé dans notre travail un test fondé sur la rapidité de développement de larves d'Oursins.

Au cours d'une série de travaux, Wilson (1951), puis Wilson et Armstrong $(1952,1954,1958)$ avaient déjà constaté qu'il était possible de distinguer des eaux de salinités voisines, mais de composition planctonique différente, d'après la vitesse de développement des larves d'Echinus esculentus. En effet, dans certaines eaux, les larves de cet Oursin, obtenues par fécondation articielle, se développaient plus lentement, parfois

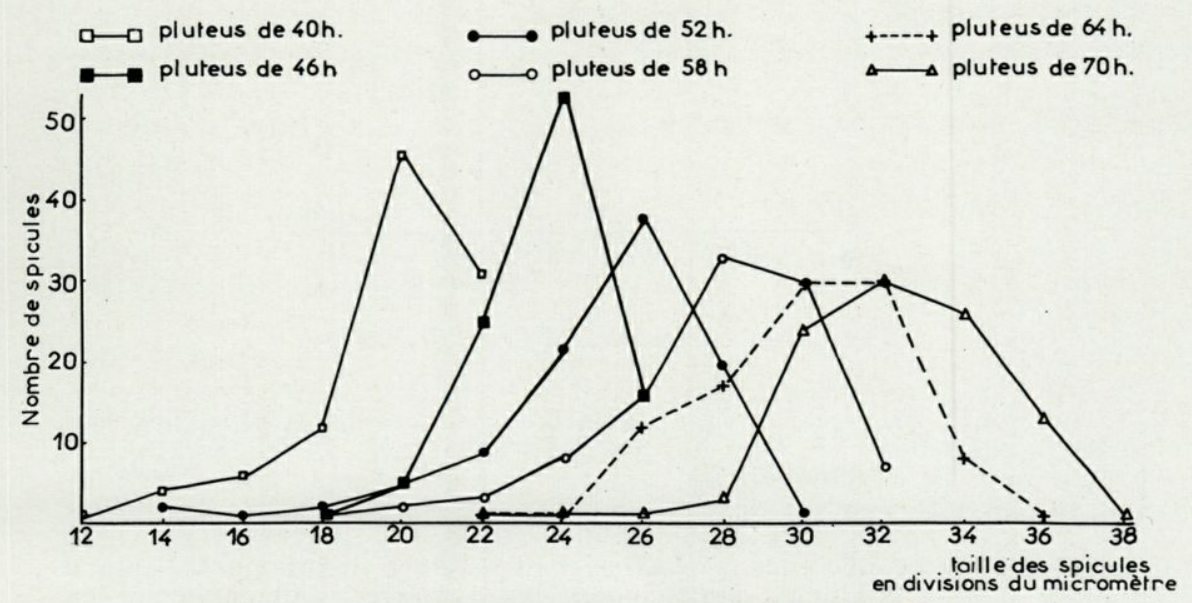

Fig. 1. - Courbes de croissance d'Arbacia lixula. 
même de façon anormale, et mourraient plus vite que dans d'autres eaux, ce qui permettait de différencier des eaux «mauvaises» et des eaux «favorables».

Dans ses travaux sur les effets biologiques du cuivre et du zinc, Bougis $(1959,1961)$ a repris ce test avec Paracentrotus lividus, en le perfectionnant. Pour mettre en évidence l'action biologique du mercure et de l'argent, nous avons employé la même méthode. Nous l'avons également adoptée avec Arbacia lixula, mais cette seconde espèce ayant un développement moins rapide que la première, il nous a fallu porter la durée des expériences de 40 heures avec Paracentrotus à 52 heures avec Arbacia, ce qui permet à celui-ci d'atteindre une taille suffisante (fig. 1) sans que la croissance se trouve ralentie (fig. 2). La technique est identique dans les deux cas et nous allons en faire ici l'exposé, en insistant sur les précautions à prendre pour assurer des conditions d'expérimentation aussi parfaites que possible.

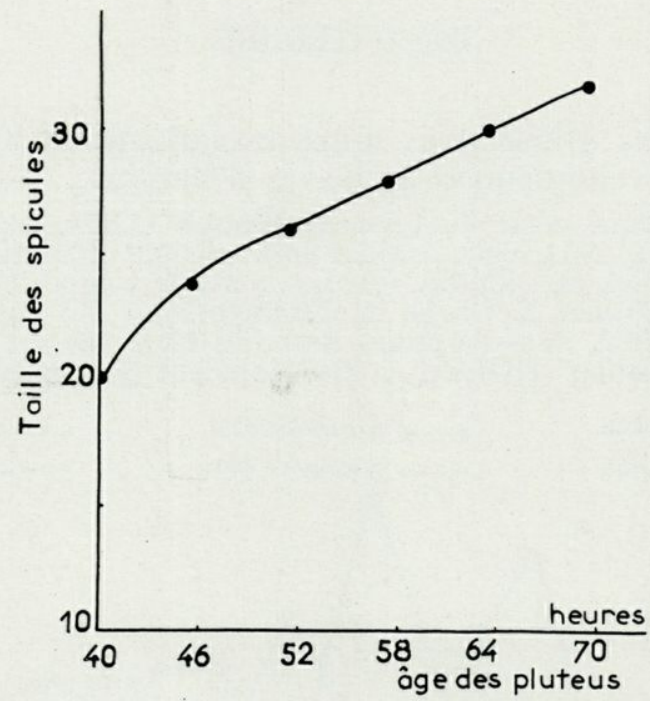

Fig. 2. - Croissance d'Arbacia lixula.

Les œufs, provenant d'une même femelle, sont lavés plusieurs fois, puis fécondés dans de l'eau de mer naturelle. Après la fécondation, un prélèvement est effectué afin de s'assurer du pourcentage d'œufs fécondés, tout lot fécondé à moins de $95 \%$ étant rejeté. Ces œufs sont alors lavés, puis répartis en quantité égale dans des boîtes cylindriques en verre, contenant $250 \mathrm{cc}$ des eaux à étudier. L'expérience ayant montré que le nombre d'œufs contenus dans chaque boîte pouvait influencer légèrement la croissance, l'optimum se situant vers 12000 œufs, un rapide 
comptage est pratiqué pour avoir une quantité d'œufs aussi voisine que possible de ce chiffre.

Ces boîtes sont ensuite placées dans un bain-marie dont la température est maintenue constante par thermostat à $20^{\circ} \mathrm{C}$. Pour rendre les conditions encore plus homogénes, les boîtes sont permutées régulièrement.

Au bout d'un certain temps, les plutéus sont rapidement filtrés, puis jetés dans l'urée $6 \mathrm{M}$ et agités. Les tissus se dissolvent et on mesure 100 à 200 spicules par lot au microscope. La mensuration s'effectue de l'extrêmité de la baguette somatique à l'extrêmité de la baguette post-orale. En attendant d'être mesurés, les spicules sont conservés au froid $\left(+2^{\circ} \mathrm{C}\right)$.

\section{LE MERCURE}

\section{Mise au poInt}

Les travaux ayant trait à l'action biologique du mercure sont plus nombreux que ceux concernant la toxicité de l'argent, $\mathrm{du}$ fait de son utilisation dans les peintures 《antifouling .

La sensibilité au mercure des Vertébrés marins est pratiquement inconnue. A notre connaissance, RICHET (1881) est le seul auteur à avoir étudié l'effet de divers sels métalliques et en particulier du mercure sur des Poissons. Il utilisait dans ses expériences des Poissons méditerranéens, Serranus cabrilla, Crenilabrus mediterraneus, Julis vulgaris et Julis gioffredi. Il constate que le chlorure mercurique est un des sels les plus toxiques et qu'une concentration supérieure à $290 \mu \mathrm{gr}$ par litre entraîne la mort des Poissons en moins de 48 heures. De ces expériences, RicheT déduit que l'action des métaux lourds dépend de leur concentration et non de leur poids atomique.

Les données concernant l'action du mercure sur les Crustacés sont plus nombreuses et mettent en évidence des variations considérables de sensibilité.

Les Cirripèdes ont été les mieux étudiés à cause du rôle qu'ils jouent dans la salissure des coques de bateaux. Pyefinch et Motr (1944-1948) montrent non seulement les différences de sensibilité qui existent entre les deux espèces Balanus crenatus et $B$. balanoides, mais encore les variations de celle-ci au cours du développement.

Au stade nauplius, $B$. crenatus se montre moins résistant que $B$. $b a$ lanoides : une concentration de 90 !̣gr par litre entraîne une mortalité de $50 \%$ chez le premier alors qu'une teneur de $230 \mu$ gr par litre provoque le même résultat chez le second. Les cypris de ces deux espèces se montrent extrêmement résistants; chez $B$. crenatus, plus sensible, une mortalité de $50 \%$ n'est atteinte qu'avec une dose de $1 \mathrm{mgr}$ par litre contre $3 \mathrm{mgr}$ par litre pour $B$. balanoides. Par contre, une très faible quantité de mercure suffit à empêcher leur fixation et le cypris de $B$. $b a$ lanoides, qui résiste à $3 \mathrm{mgr}$ de mercure par litre, ne peut plus se fixer dès que la concentration atteint $50 \mu \mathrm{gr}$ par litre. 
Les adultes de $B$. crenatus se montrent plus résistants que ceux de $B$. balanoides, contrairement à ce qui se passait pour les larves. De plus, chez cette espèce, la sensibilité diminue avec l'âge. Une mortalité de $50 \%$ n'est obtenue qu'avec $1,35 \mathrm{mgr}$ par litre contre $360 \mu \mathrm{gr}$ par litre pour B. balanoides.

Ces résultats ont été retrouvés par Clarke (1947) chez Balanus eburneus et $B$. balanoides; cet auteur montre également que le stade cypris est le plus résistant. En effet la concentration de 16,6 mgr par litre nécessaire pour empêcher la fixation des larves est bien supérieure à celle supportée par les adultes. Ces variations de sensibilité au cours du développement paraissent donc courantes chez les Cirripèdes.

Les renseignements que nous possédons sur les autres Crustacés sont peu nombreux. Hunter (1949) étudie et compare les actions du cuivre et du mercure sur un Amphipode, Marinogammarus marinus. Malheureusement il utilise un test de toxicité basé sur l'activité de l'animal, ce qui rend ses données peu comparables avec celles des autres auteurs. Il ressort de ses résultats qu'une quantité de 2,5 $\mu$ gr de mercure par litre se montre très toxique pour Marinogammarus marinus.

Les travaux de Barnes et Stanbury (1948) sur Nitocra spinipes et de Corner et Sparrow $(1956,1957)$ sur Artemia salina, Elminius modestus et Acartia clausi, sont à rapprocher, car ils permettent de mettre en évidence l'influence du mode de vie sur la résistance des différentes espèces. Barnes et Stanbury constatent qu'une mortalité de $50 \%$ au bout de 24 heures est atteinte avec Nitocra spinipes dans une eau à teneur en mercure de $600 \mu \mathrm{gr}$ par litre. Avec Acartia clausi, Corner et Sparrow obtiennent le même résultat après un séjour de 2 heures 30 dans une eau à $50 \mu \mathrm{gr}$ de mercure par litre. Cette différence de sensibilité est à mettre en relation avec le fait que le premier est un Copépode Harpacticide vivant dans des eaux plus côtières donc plus polluées, alors que le second est un Copépode planctonique de haute mer.

De même, Corner et Sparrow mettent en évidence la très grande tolérance vis-à-vis du mercure des larves d'Artemia salina, espèce bien connue pour la faculté qu'elle a de s'adapter à des variations chimiques de grande amplitude : au bout de 2 heures 30, une mortalité de $50 \%$ n'est atteinte qu'avec une concentration de $800 \mathrm{mgr}$ de mercure par litre. Les larves d'Elminius modestus se montrent beaucoup plus sensibles et $30 \mu$ gr par litre suffisent pour obtenir le même résultat.

L'action du mercure sur les Echinodermes n'a été étudiée que pendant les premiers stades du développement. Lillie (1921), HoAdley (1923, 1930) étudient l'action de certains sels, dont le chlorure mercurique, sur la réaction de fertilisation et les premières phases de la segmentation d'Arbacia aequituberculata. Ils constatent que le chlorure mercurique n'empêche nullement la fécondation et favorise même l'élévation de la membrane de fertilisation; celle-ci peut être provoquée sur des œufs non fécondés par l'addition de fortes quantités de sel. Par contre une faible concentration en mercure suffit pour entraîner des troubles dans la segmentation, un retard dans le développement et une diminution de la viabilité du zygote.

Waterman (1937) étudie également l'action inhibitrice de plusieurs sels de métaux lourds sur le développement d'Arbacia punctulata. Le chlorure mercurique se montre l'un des plus toxiques bien que n'affectant nullement la fécondation et la réaction de fertilisation. La sensibilité de l'œuf semble augmenter avec les progrès du développement et une concentration de $15 \mu \mathrm{gr}$ de mercure par litre suffit à le retarder. L'action du mercure est très rapide car des œufs plongés 5 minutes seulement dans une eau de mer a laquelle $290 \mu \mathrm{gr}$ de mercure par litre ont été ajoutés ont leur croissance retardée de 24 heures. 
Il est intéressant de remarquer que le mercure ne semble pas avoir le même mode d'action que les autres métaux lourds, en particulier le cuivre. En effet son action préalable ou simultanée provoque une augmentation des effets de ce dernier.

Barnes et Stanbury avaient constaté le fait qu'un mélange cuivremercure se montrait beaucoup plus toxique pour Nitocra spinipes que l'addition des effets respectifs ne le faisait supposer. Hunter retrouve ce résultat avec Marinogammarus marinus. Il observe en plus qu'un animal déjà attaqué par le mercure se trouvait sensibilisé au cuivre alors que la réciproque n'était plus vraie. CoRner et Sparrow reprennent ces expériences avec Artemia salina et constatent les mêmes effets du mercure.

Ce métal aurait donc une action différente de celle du cuivre. Il agirait directement sur le protoplasme alors que le cuivre modifierait le métabolisme, ce qui expliquerait que seul ce dernier provoque une inhibition de la respiration.

Dans une publication plus récente (1957), les mêmes auteurs ont montré que les effets biologiques du mercure pouvaient être diminués dans une forte proportion par l'action de complexants comme le B.A.L., la cystéine, le gluthation et l'acide thioglycollique. Le cas est d'ailleurs général avec les métaux lourds.

\section{EFFEts biologiques dU MERcure SUR LE DÉvelopPEMENT DE} PARACENTROTUS LIVIDUS

L'eau de mer a été récoltée en surface à l'entrée de la rade de Villefranche, dans une bonbonne en polyéthylène. Cette eau, filtrée sur papier, nous a servi comme référence. Nous lui avons ajouté des doses de mercure $\left(\mathrm{HgCl}_{2}\right)$ variant de 1 à $10 \mu \mathrm{gr}$ par litre et nous en avons observé les effets.

Les résultats obtenus le $3 / 2 / 60$ nous ont montré que l'addition de $10 \mu \mathrm{gr}$ de mercure par litre provoquait une très grande perturbation dans le développement. Il s'est révélé impossible d'établir une courbe de fréquence, vu le petit nombre de plutéus que nous avions obtenus. Le squelette de ces derniers, de taille très réduite, était déformé.

Lors de la même expérience, une quantité de 5 ugr par litre a entraîné un retard très net dans le développement; de même une dose de 3 ugr par litre entraîne un décalage sensible de la courbe de fréquence. Par contre des quantités de 2 et 1 ugr par litre se sont montrées sans effet.

Ces résultats ont été portés sur la figure 3, A. Les courbes de fréquence obtenues avec 2 et $1 \mu \mathrm{gr}$ par litre sont comparables à celle des témoins. La courbe obtenue avec $3 \mu$ gr par litre est légèrement décalée. Enfin, les plutéus élevés dans une eau additionnée de $5 \mu \mathrm{gr}$ par litre se montrent très attardés.

L'expérience du $1 / 3 / 60(5,4,3,2$ et $1 \mu$ gr par litre) a confirmé ces résultats et nous a permis de mettre en évidence l'exis- 
tence d'un seuil de toxicité situé entre 2 et $3 \mu \mathrm{gr}$ par litre. En effet, les plutéus obtenus dans une eau de mer additionnée de 3 ugr par litre avaient une longueur moyenne de 38,58 divisions du micromètre $(389 \mu)$ contre 39,34 pour les témoins $(397 \mu)$. La comparaison statistique des deux moyennes donne pour $t$ une valeur de 2,45 significative $(\mathrm{P}<0,05)$. Bien que cette valeur de $t$ ne soit que significative, il semble bien que le seuil de toxicité se situe autour de $3 \mu \mathrm{gr}$ par litre, car les courbes de fréquence des lots élevés dans la même eau à laquelle nous avions ajouté 1 et $2 \mu \mathrm{gr}$ par litre, ont été identiques à celle du lot témoin.
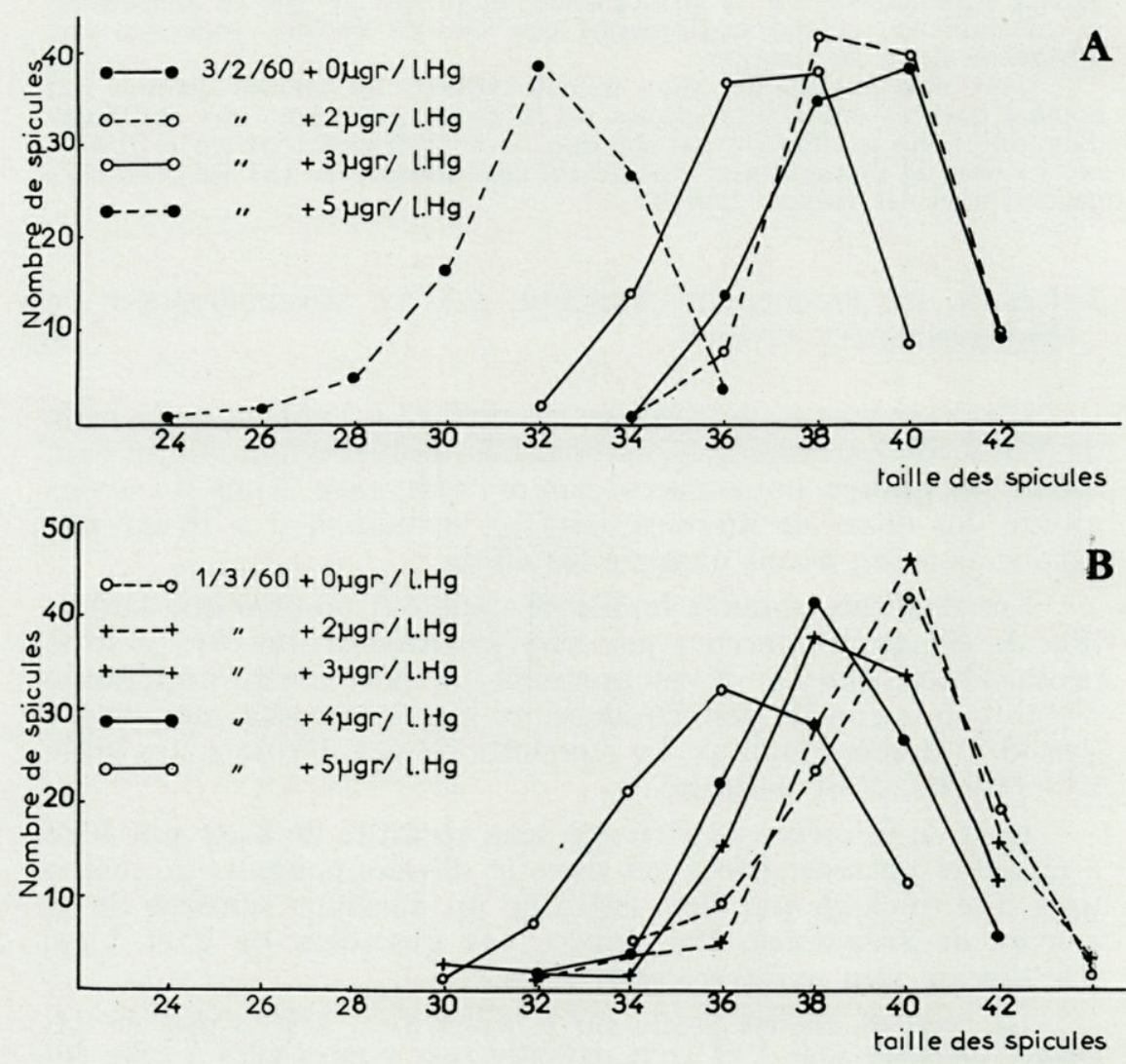

Fig. 3. - Effets du mercure sur la croissance de Paracentrotus lividus.

Nous n'avons porté sur la figure 3 , B que les courbes obtenues pour $5,4,3$ et $2 \mu$ gr de mercure par litre, la courbe de fréquence correspondant à $1 \mu \mathrm{gr}$ par litre étant comparable à cette dernière. 


\section{Conclusions}

Les expériences que nous avons réalisées nous ont montré que le mercure n'était toxique pour les larves de Paracentrotus lividus qu'à des doses relativement importantes : pour obtenir une diminution de la longueur des spicules décelable par notre test, une concentration de $3 \mu \mathrm{gr}$ de métal par litre est nécessaire. Or la seule valeur que nous possédons concernant sa concentration dans l'eau de mer nous indique une quantité de mercure de $0,03 \mu$ gr par litre (STOck et Cucuel à Heligoland, 1934). Il est cependant permis de penser que des analyses ultérieures montreront que la teneur en mercure peut atteindre des valeurs plus élevées dans les eaux côtières, plus polluées, ou au contact du sédiment. Dans l'état actuel de nos connaissances, le mercure ne jouerait, du moins en ce qui concerne les larves d'Oursin, qu'un rôle réduit bien que, comme nous l'avons vu, il puisse renforcer l'action d'autres métaux lourds.

\section{L'ARGENT}

\section{MISE AU POINT}

Les données que nous possédons concernant l'action biologique de l'argent dans le domaine marin sont rares et manquent de précision. De plus l'action toxique de l'argent a été fortement controversée pendant longtemps : c'est ainsi que les résultats obtenus par RAulin (1887) avec des cultures d'Aspergillus furent critiqués et l'année 1913 vit paraître 3 notes successives de CoDur, de Clément et de Sauton qui arrivaient, avec le même matériel, à des conclusions variant dans de larges proportions et, parfois, contradictoires.

A notre connaissance, un seul auteur, LARoze (1955), a étudié l'action des sels d'argent, sur Squalus cephalus. L'auteur constate que la toxicité de l'argent est très importante et qu'elle varie avec la température.

Il démontre que l'action toxique des métaux lourds sur les branchies de Poissons, n'est pas dans la coagulation du mucus qui les recouvre, mais dans une action plus intime sur le protoplasme des cellules, avec blocage enzymatique. Ce serait donc une véritable intoxication. Au-dessus de $15^{\circ} \mathrm{C}$, l'action de l'argent augmente de facon sensible avec la température. La température de $15^{\circ} \mathrm{C}$, à laquelle la plupart des métaux ont des effets toxiques réduits, doit être en rapport avec un maximum d'activité physiologique du Poisson, et par conséquent avec une désintoxication plus rapide, comme cela se passe avec les insecticides.

Les données concernant la sensibilité des divers Crustacés ne sont 
ni plus nombreuses ni plus précises. Clarke (1947) constate que le sulfate d'argent est un des sels les plus toxique pour Balanus eburneus et $B$. balanoides. En effet, $400 \mu$ gr d'argent par litre entraînent la mort de $90 \%$ des adultes de $B$. balanoides en 48 heures. Croghan (1958) étudie la résistance d'Artemia salina au nitrate d'argent. Il démontre que, contrairement à ce qu'avaient écrit Boone et BAAS Becking (1931) ce sel est extrêmement toxique pour Artemia salina et qu'une concentration de $1 \mathrm{mgr}$ d'argent par litre entraîne la mort au bout d'une vingtaine de minutes.

Les œufs, le sperme et les larves de Paracentrotus lividus, des Planaires du genre Convoluta, ont été utilisés par Drzewina et BoHN (1921 à 1931) lors de leurs recherches sur la nature physique ou chimique de l'action de l'argent.

Si les données fournies par ces deux auteurs manquent de précision surtout quant aux doses employées, elles sont néanmoins intéressantes, car elles nous donnent une idée de la complexité du problème envisagé.

DRZEwINA et BoHN constatent tout d'abord une action immédiate de l'argent; des plutéus nageant, placés dans une coupelle en argent, sont immédiatement paralysés, puis cytolisés en moins de 10 minutes. Les Convoluta, arrivant au contact d'une pièce de monnaie, se coupent en deux et se cytolisent. A notre avis, ces effets brutaux devaient être en rapport avec l'importante concentration en métal et le faible volume utilisés.

Ces deux auteurs mettent en évidence des effets plus tardifs. Trempant des œufs dans de l'eau d'argent (eau obtenue en faisant séjourner de l'eau de mer dans un récipient en argent), ils constatent qu'un bain de quelques secondes (parfois 2 secondes) suffit à empêcher le développement et à provoquer la cytolyse au bout d'un temps variable. Ils montrent l'influence du nombre d'individus en expérience sur la résistance de ces individus. Ils constatent également que la lumière agit de faccon sensible sur l'action toxique de l'argent. Bien avant Holm JENSEN (1948), ils observent l'action antagoniste à celle de l'argent, de certains acides aminés tels que le glycocolle et l'alanine. Ils remarquent également l'action antagoniste que l'étain exerce sur l'inhibition par l'argent de la calcication du plutéus.

\section{L'ARgENT DANS L'EAU DE MER}

La présence de l'argent dans l'eau de mer est connue depuis fort longtemps. Dès 1787, Proust, dans une lettre adressée à LA Metherie, qui la publia dans le Journal de Physique de la même année, déclare qu'il croit à l'existence dans l'eau de mer de l'argent et d'autres métaux.

Dans une note publiée en $\mathbf{1 7 9 9}$ dans le même périodique, où il affirme la présence du mercure dans l'eau de mer et dans le sel marin, l'illustre chimiste indique un moyen ingénieux de vérifier ses affirmations, moyen basé sur l'action réductrice qu'une lame de cuivre exerce sur le chlorure d'argent dissous dans le chlorure de sodium : «Si quelqu'un, après avoir lu ceci, prenait la peine d'observer si le doublage d'un vaisseau nouvellement mis à la mer s'argentait dans quelque partie, surtout lorsqu'il commence à sillonner pour la première fois les mers; s'il prenait la peine de suspendre dans leurs eaux une plaque d'or pour en observer les changements, il pourrait se flatter peut-être de fournir, à son retour, 
un article de plus à l'histoire naturelle du sel marin ? Qui sait si la destruction des doublages, quelquefois si rapide, et encore inconnue dans sa cause, ne dépendrait point de l'existence du mercure, plus abondant dans certaines mers que dans d'autres.»

Tuld confirme par cette méthode l'existence de l'argent dans l'eau de mer, existence constatée par Malaguti, Durocher et SARzeau (1850) dans l'eau de la Manche, auteurs auxquels on doit la première estimation quantitative $(0,00001 \mathrm{gr} / 1)$.

Tuld constate en effet que le cuivre du doublage d'un bateau ayant navigué pendant sept ans dans le Pacifique, contenait $0,5 \%$ d'argent. En comparant un doublage en cuivre neuf avec un doublage ayant servi pendant trois ans dans le Pacifique, cet auteur constate que le second contenait huit fois plus d'argent que le premier (Globe 14/1/1857).

Forchнамmer (1865) confirme l'existence de l'argent dans les eaux de la Baltique. Mais à notre connaissance, trois publications seulement font état de valeurs précises concernant la teneur en argent des différentes mers.

Les travaux de HABER (1927), réalisés avec des moyens très importants, sont de loin les plus complets. Cet auteur, qui s'intéressait surtout à l'or, a en effet analysé de très nombreux échantillons de provenances variées mais, comme il l'indique luimême, ses données sont relativement peu précises en dépit des précautions observées. Elles sont cependant intéressantes par leur nombre et par l'idée qu'elles donnent des variations de la teneur en argent suivant les lieux et la profondeur, variations que nous allons tenter de mettre en évidence à partir des résultats bruts fournis par HABER.

En ce qui concerne le Pacifique, les échantillons récoltés à l'entrée de la baie de San Francisco présentent des concentrations en argent variant de 0,01 à $0,29 \mu \mathrm{gr}$ par litre, la valeur moyenne se situant aux environs de $0,07 \mu \mathrm{g}$. Mais dans les échantillons récoltés à l'intérieur de la baie, près du dẻbarcadère d'Oakland, cet auteur trouve des quantités d'argent nettement supérieures, de 0,01 à $3,10 \mu g r$ par litre, la moyenne oscillant autour de 0,400 $\mu \mathrm{gr}$. La teneur en argent, comme c'est d'ailleurs le cas pour la plupart des métaux lourds, augmente de façon sensible au voisinage des côtes.

En Atlantique, la concentration en argent est plus forte et les teneurs varient autour d'une moyenne de $0,300 \mu \mathrm{gr}$ par litre $(0,04<\mathrm{C}<4,60 \mu \mathrm{gr}$ /1). Ces résultats nous ont également permis de constater que la quantité d'argent contenu dans l'eau de mer diminuait avec la profondeur : à la station 11 (campagne du «Meteor»), alors que la concentration en argent des eaux de surface est de 1,60 $\mu \mathrm{gr}$ par litre, elle n'est plus que de $0,1 \mu \mathrm{gr} / \mathrm{l}$ à $700 \mathrm{~m}$, pour atteindre $0,04 \mu \mathrm{gr}$ à $2500 \mathrm{~m}$. Si, pour les autres stations, la baisse de concentration ne se fait pas toujours de façon aussi régulière, le phénomène est cependant remarquablement constant.

Il se retrouve d'ailleurs dans les eaux arctiques (campagne de la 《Dana ») qui présentent des teneurs en argent plus fortes que celles de l'Atlantique et du Pacifique. Elles descendent en effet très rarement en dessous de 0,100 $\mu$ gr par litre et, en surface, la teneur moyenne est de 
$0,400 \mu$ gr par litre, alors qu'à $400 \mathrm{~m}$, elle s'établit autour de $0,120 \mu$ gr. Les échantillons récoltés plus au Nord par le "Godthaab» montrent des concentrations tout à fait comparables, mais il semble que la diminution observée avec la profondeur soit ici en rapport avec l'augmentation de la salinité, l'influence de l'eau provenant de la fonte des glaces à très forte concentration en argent (1 $\mu \mathrm{gr}$ par litre) se faisant sentir essentiellement sur les eaux de surface.

Plus récemment Noddack et Noddack (1940), se basant sur une seule analyse pratiquée sur un échantillon d'eau de surface prélevé dans le Gullmarfjord, ont évalué la concentration en argent à $0,15 \mu \mathrm{gr}$ par litre.

Dans un travail paru en 1952, BLACK et Mrtchell indiquent, pour des eaux prises au large de Plymouth, des teneurs de 2,9 et $2,1 \mu \mathrm{gr}$ par litre, et inférieures à $0,4 \mu \mathrm{gr}$ pour des échantillons récoltés à Ardencaple Bay et Atlantic Bridge. Ces résultats sont en contradiction avec ceux que Haber a obtenu à San Francisco, mais ces auteurs insistent sur le fait qu'ils travaillent à la limite des possibilités de la méthode employée et que leurs résultats sont sujets à caution.

Il nous faut rappeler les travaux de Lizunov et Lisitsyn (1955) sur la répartition et la composition des éléments en suspension de la mer de Berhing, bien que les données de ces auteurs ne correspondent qu'à l'argent sestonique. Ces données ne sont d'ailleurs pas numériques, mais elles permettent de faire d'intéressantes comparaisons. Lizunov et LisitsYN observent, en effet, une concentration exceptionnelle de l'argent au printemps et en automne dans certaines parties du littoral et notamment à l'embouchure des rivières Anadyr' et Kamtchatka, mais ils attribuent celle-ci à la présence de gîtes métallifères sous-marins et côtiers et non à l'apport des cours d'eau, dont la teneur en argent est très faible.

Mais, comme l'indique Richards (1956), toutes ces valeurs, obtenues par des méthodes différentes, ne sont pas comparables et il faut bien se garder d'en tirer des conclusions générales.

$\mathrm{Au}$ cours de notre travail, nous avons dû mettre au point une méthode simple d'analyse quantitative de l'argent dans l'eau de mer de façon à pouvoir préciser la valeur du seuil de toxicité de ce métal.

\section{Analyse quantitative de l'argent}

Comme nous l'avons vu dans l'historique, les données concernant la teneur en argent des différentes mers sont rares. C'est que le dosage de l'argent se heurte non seulement aux difficultés communes à l'analyse quantitative des autres oligoéléments, contamination possible des échantillons et mauvaise conservation de ces échantillons, mais aussi au fait qu'il n'existe aucun réactif véritablement spécifique de ce métal. 
Les deux réactifs les plus employés en colorimétrie, sont la p-diéthyl ou p-diméthylaminobenzylidènerhodanine et la diphénylthiocarbazone ou dithizone; nous avons utilisé cette dernière au cours de notre travail.

a) Caractéristiques de la dithizone et de ses réactions :

La dithizone, de formule

$$
\mathrm{S}=\mathrm{C} \nearrow_{\mathrm{N}=\mathrm{N}-\mathrm{C}_{6} \mathrm{H}_{5}}^{\mathrm{NH}-\mathrm{NH}-\mathrm{C}_{6} \mathrm{H}_{5}}
$$

est un solide noir violet, soluble dans la plupart des solvants organiques. En analyse, sont utilisés le tétrachlorure de carbone et le chloroforme; nous nous sommes servi de ce dernier. La solution obtenue est dichroïque, rouge en lumière transmise et verte en lumière réfléchie.

Lorsqu'une telle solution est agitée avec une solution aqueuse de métal lourd, il y a formation d'un dithizonate primaire existant pour tous les métaux lourds et se formant, en général, en milieu acide.

La réaction, réversible est du type :

$$
2 \mathrm{H} \mathrm{Dz}+\mathrm{Me}^{++} \longrightarrow \mathrm{Me} \mathrm{Dz}{ }^{2}+2 \mathrm{H}^{+}
$$

où $\mathrm{Me}^{++}$est un métal bivalent.

Dans ce cas, la constante d'équilibre de la réaction est :

$$
\mathrm{k}=\frac{\left(\mathrm{MeDz}^{2}\right)_{w}\left(\mathrm{H}^{+}\right)_{w}^{2}}{\left(\mathrm{Me}^{++}\right)_{w}(\mathrm{HDz})_{w}^{2}}
$$

En présence d'une phase aqueuse et d'une phase organique, cette constante d'équilibre devient :

$$
\frac{\left(\mathrm{MeDz}^{2}\right)_{0}\left(\mathrm{H}^{+}\right)_{w}^{2}}{\left(\mathrm{Me}^{++}\right)_{w}(\mathrm{HDz})_{0}^{2}}=\mathrm{K}
$$

dans laquelle $o$ et $\mathrm{w}$ désignent la phase organique et la solution aqueuse. En effet, les coefficients de distribution de la dithizone et du complexe métallique entre l'eau et le solvant organique sont :

$$
\mathrm{D}_{\mathrm{HOz}}=\frac{(\mathrm{HDz})_{w}}{(\mathrm{HDz})_{0}} \quad \text { et } \quad \mathrm{D}_{\text {MeOz }}=\frac{\left(\mathrm{MeDz}^{2}\right)_{w}}{\left(\mathrm{MeDz}^{2}\right)_{0}}
$$

A partir de l'équation 3 , on tire le coefficient d'extraction du métal :

$$
\frac{\left(\mathrm{MeDz}^{2}\right)_{0}}{\left(\mathrm{Me}^{++}\right)_{w}}=\mathrm{K} \frac{\left(\mathrm{H}^{2} \mathrm{Dz}\right)_{0}^{2}}{\left(\mathrm{H}^{+}\right)_{0}^{2}}
$$


Le coefficient $K$, ou constante d'extraction est en général obtenu par expérimentation directe. De cette dernière équation il ressort que l'extractibilité du métal à un $\mathrm{pH}$ donné augmente avec le carré de l'excès de dithizone dans la phase organique, ou encore qu'avec un excès de dithizone constant, elle est inversement proportionnelle au carré de la concentration en $\mathrm{H}^{+}$.

En milieu basique ou déficitaire en dithizone, pour certains métaux comme le cuivre, l'argent et le mercure, il y a formation d'un dithizonate secondaire d'après la réaction réversible :

$$
\mathrm{Me} \mathrm{Dz}^{2}+\mathrm{Me}^{++} \longrightarrow 2 \mathrm{Me} \mathrm{Dz}
$$

Les composés métalliques, solubles en général dans le solvant organique, présentent des couleurs extrêmement vives, violet, jaune, orangé, rouge. C'est ainsi que l'argent, en milieu acide et en présence d'un léger excès de dithizonate, réagit pour donner avec cette dernière un dithizonate primaire d'argent, de couleur jaune, $\mathrm{Ag} \mathrm{Dz}$, soluble dans le tétrachlorure de carbone et le chloroforme (fig. 4). En milieu neutre ou basique, il y a formation du dithizonate secondaire, $\mathrm{Ag}_{2} \mathrm{Dz}$, de couleur violacée,

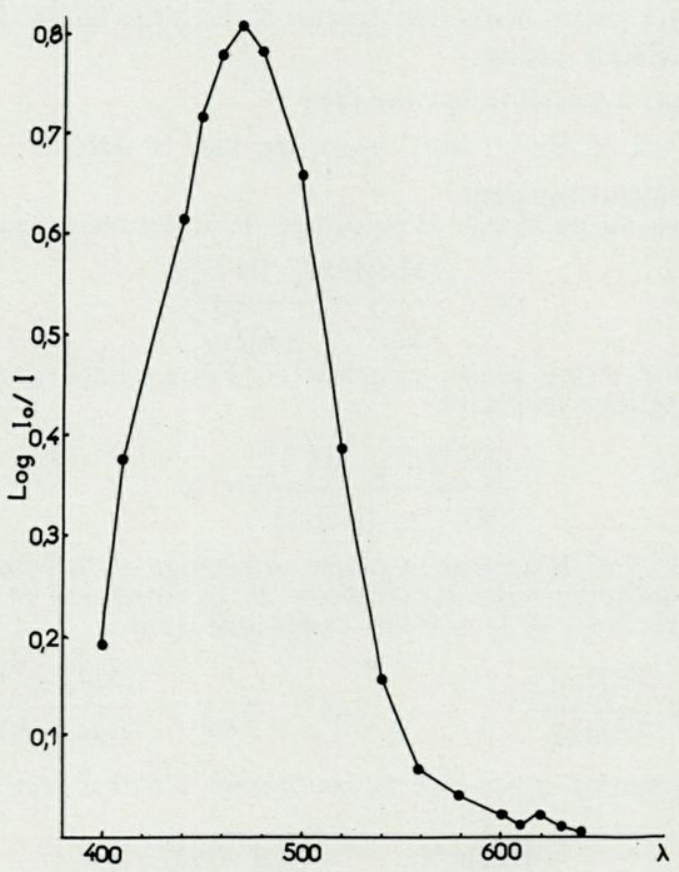

Fig. 4. - Spectre d'absorption du dithizonate primaire d'argent. 
pratiquement insoluble, et qui ne présente aucun intérêt en analyse.

L'intensité et la vitesse de réaction dépendent d'un certain nombre de facteurs tels que la concentration en dithizone, la présence de complexants masquant les métaux, le rapport entre le volume de la phase aqueuse et celui de la phase organique contenant le réactif, et surtout le $\mathrm{pH}$ et le temps d'agitation.

En effet, si en milieu basique la plupart des métaux sont extraits rapidement et complètement, en milieu acide, il n'en est plus de même. Les métaux à constante d'extraction importante comme le palladium, l'argent, le mercure, sont extraits beaucoup plus vite que ceux à petite constante tels que le cuivre; le zinc et le plomb nécessitent pour leur extraction un milieu faiblement acide et même basique. Ce fait est important en analyse car il permet d'orienter la réaction vers un métal défini et de la rendre ainsi spécifique. Or le problème le plus important posé par l'eau de mer provient du fait que nous y trouvons en quantité variable, du bismuth, du cobalt, du cuivre, du zinc, de l'étain, du fer, de l'or, du mercure, du manganèse, du nickel et de l'argent, métaux qui forment tous des composés avec la dithizone.

WichMaNN (1939), KoROLEFF (1950), WolfF (1954), ont pu établir des séries de courbes mettant en évidence les relations entre le $\mathrm{pH}$ de la phase aqueuse et la complète extraction des différents métaux. Ces courbes sont fonctions des conditions dans lesquelles elles ont été obtenues, ce qui explique les variations observées d'un auteur à l'autre et notamment par WoLFF.

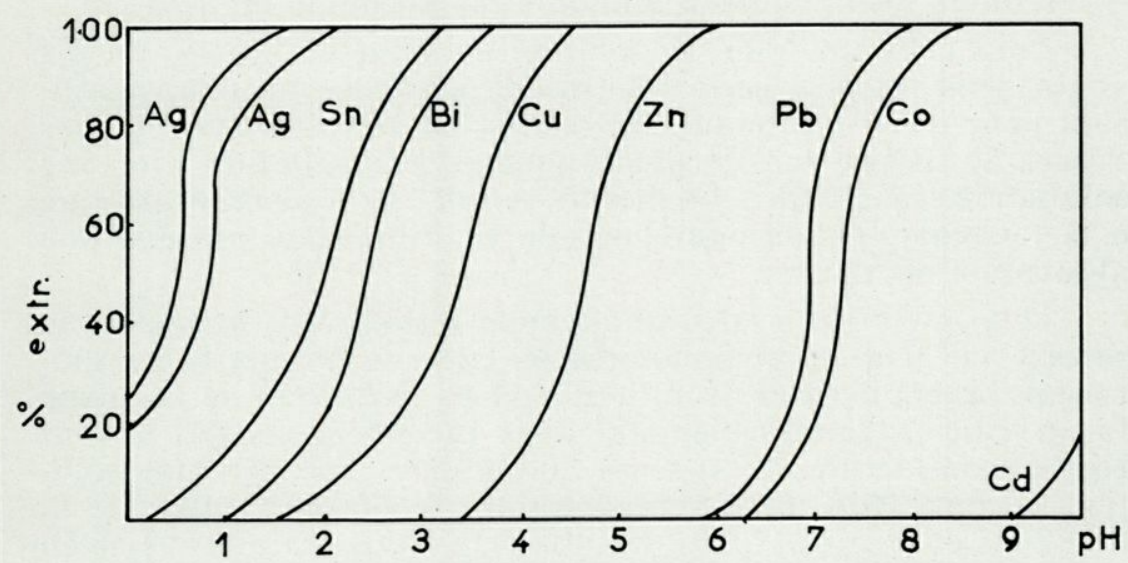

Fig. 5. - Pourcentage d'extraction des différents métaux par la dithizone en fonction du $\mathrm{pH}$ (d'après Wichmans, revu par WolfF).

D'après KorolefF, à $\mathrm{pH}$ très acide $(\mathrm{pH} 2)$, une agitation de cinq minutes est suffisante pour obtenir une extraction complète. D'autre part, d'après les courbes du même auteur et celles de WolfF (fig. 5), à un tel $\mathrm{pH}$, l'argent serait pratiquement le seul métal à entrer en combinaison avec la dithizone. 
Mais comme l'indique KorolefF :

- en pratique, une élimination totale des interférences par le $\mathrm{pH}$ n'est pas possible et il est nécessaire de faire agir les complexants.

- les réactions sont plus longues dans le chloroforme que dans le tétrachlorure de carbone.

- certains anions comme les cyanures, les citrates, les tartrates, ont également une influence sur les réactions qui diffère d'un métal à l'autre. En effet, les métaux forment avec eux des complexes plus forts que ceux formés avec la dithizone.

- Enfin, et surtout en eau de mer océanique, à plus forte raison en Méditerranée, où la salinité est forte, l'extraction doit se faire à un $\mathrm{pH}$ plus important que la constante d'équilibre ne le laisse prévoir.

Ce dernier fait a été mis en évidence par Friedeberg (1955) qui constate que si, en l'absence de chlorures, la totalité de l'argent est extraite à $\mathrm{pH} 2$, le $\mathrm{pH}$ doit être porté à 3,5 pour une teneur en chlorures supérieure à $1 \%$ et à 5 pour une teneur de $20 \%$.

\section{b) Méthodes d'analyse :}

En l'absence de toute méthode d'analyse spécialement adaptée à l'eau de mer, nous avons utilisé les méthodes classiques, en essayant d'éliminer les interférences dues aux autres métaux. Ces méthodes sont de plusieurs types et nous allons les passer en revue.

- Méthode de la couleur unique («monocolour method»).

Dans cette méthode, la solution aqueuse est ajustée au $\mathrm{pH}$ voulu, puis agitée avec la solution de dithizone un temps suffisant pour que l'extraction soit complète. On fait alors repasser l'excès de réactif dans la phase aqueuse par agitation avec une solution basique diluée. La mesure se fait sur la phase organique à la longueur d'onde pour laquelle le dithizonate possède une absorption maximum.

Cette méthode n'est pratiquement utilisée que lorsqu'on ne se sert pas d'un spectrophotomètre, car elle permet la comparaison directe avec des solutions étalons préparées de la même façon. Elle présente cependant deux inconvénients qui la font rejeter dans la plupart des cas : d'une part, l'usage d'une solution basique trop forte peut entraîner la décomposition de la dithizone et même la transformation du dithizonate primaire en dithizonate secondaire; d'autre part, si au contraire la solution est trop faible, l'élimination de la dithizone en excès n'est pas totale.

- Méthode des couleurs superposées («mixed colour method»). Réactif : dithizone.

Ici, l'excès de réactif est laissé dans la phase organique, mais la mesure se fait au moyen d'un spectrophotomètre, soit 
à la longueur d'onde à laquelle l'absorption du dithizonate formé est maximale (ici $470 \mathrm{~m} \mu$, et l'on observera alors une augmentation de l'absorption proportionnelle à la quantité de dithizonate formé), soit à la longueur d'onde correspondant au maximum d'absorption de la dithizone $(620 \mathrm{~m} \mu$ et l'on observera alors une diminution de l'absorption correspondant à la quantité de dithizone transformée en dithizonate, diminution évidemment proportionnelle à la quantité de métal fixé).

Mais pour que les résultats soient en accord avec la loi de Beer, il est nécessaire que la lumière soit monochromatique et la dithizone suffisamment en excès.

Nos essais de dosage de l'argent par cette méthode, en utilisant la dithizone comme réactif, se sont montrés négatifs, bien que nous ayons abaissé le $\mathrm{pH}$ de l'eau de mer à 4 , valeur minimale de $\mathrm{pH}$ à laquelle peut se faire l'extraction. La réaction n'est pas spécifique.

Le cuivre notamment est extrait à ce $\mathrm{pH}$. Présent dans l'eau de mer à une concentration de l'ordre de $1 \mu \mathrm{gr} / \mathrm{l}$, ce ne serait pas le seul métal à être extrait et, en se basant sur les constantes d'extraction, le mercure et l'or pourraient également produire des interférences; celles-ci seraient moins importantes car les teneurs naturelles de l'or (HABER, $1927: 0,004 \mu \mathrm{gr} / \mathrm{l}$ ) et du mercure (Stock et Cucuel, $1934: 0,03 \mu \mathrm{gr} / \mathrm{l}$ ) sont faibles.

Nous avons laissé de côté la méthode préconisée par KoRoLEFF en conclusion à sa thèse pour éliminer les dithizonates de cuivre et de mercure, car elle nécessite l'emploi de nombreuses solutions et des manipulations multiples; or le chloroforme étant légèrement soluble dans l'eau, chaque manipulation et agitation en présence d'une solution aqueuse provoque, tout en augmentant les risques de contamination, une concentration de la fraction organique qui rend délicate l'interprétation des résultats.

- Méthode de la réversion.

Cette troisième méthode consiste à faire agir la solution de réactif sur la solution aqueuse de métal, puis à faire une mesure à la longueur d'onde correspondant au maximum d'absorption de la dithizone, longueur d'onde à laquelle les dithizonates n'ont qu'une faible absorption. Ensuite on fait agir un complexant du métal à analyser. Le dithizonate de ce métal est ainsi décomposé, libérant la fraction de dithizone combinée et provoquant alors une concentration de la solution proportionnelle à la quantité de métal. Il devient même possible d'extraire à nouveau le métal de la phase aqueuse et de le doser directement.

L'edta, d'après HaRa (1958), complexerait tous les métaux 
autres que l'argent. Pour notre part, nous avons essayé la méthode de la réversion d'Irving et Andrew (1949). Ces deux auteurs utilisent, pour la réversion du dithizonate primaire d'argent, l'iodure de potassium, qui forme avec l'argent un complexe stable en libérant la dithizone.

Nos essais nous ont montré que dans le cas de l'eau de mer la réaction n'était pas spécifique, l'iodure de potassium provoquant la réversion d'autres dithizonates que celui de l'argent. Aussi n'avons-nous pas persisté.

Le cuivre étant le principal agent d'interférence, nous en sommes venus à utiliser le dithizonate de cuivre comme réactif, ce qui élimine presque totalement l'influence de ce métal.

- Méthode des couleurs superposées (mixed-colour method). Réactif : dithizonate de cuivre.

Une erreur négative peut apparaître dans l'estimation quantitative de l'argent avec ce réactif dans le cas où le cuivre est présent en proportions relativement importantes. D'après SANDELL (1959), en présence de 2,5 mgr de cuivre, l'erreur serait de 0,0005 pour 0,010 mgr d'argent. Dans le cas de l'eau de mer son influence peut donc être considérée comme négligeable.

L'emploi du dithizonate de cuivre éliminant en principe tout danger d'interférence, l'utilisation d'un $\mathrm{pH}$ très acide ne se justifie plus. Le fait de ne pas ajouter de tampon à l'eau de mer, donc de ne pas modifier son acidité, présente l'avantage de limiter les dangers de contamination et de travailler sur de l'eau aussi peu modifiée que possible.

Cependant, travailler au $\mathrm{pH}$ de l'eau de mer, donc en milieu alcalin ( $\mathrm{pH} 8,2$ ), ne va pas sans inconvénient, du moins du point de vue théorique :

- le dithizonate de cuivre primaire doit se transformer en dithizonate de cuivre secondaire de couleur jaune, qui va venir masquer la coloration due au dithizonate d'argent;

- à la place du dithizonate primaire d'argent, seul dosable en colorimétrie, doit se former le dithizonate secondaire, de couleur violacé et insoluble dans le chloroforme.

En pratique, dans les conditions de nos expériences, aucune de ces deux transformations ne s'est manifestée.

Nous avons réalisé quelques expériences destinées à mettre en évidence la spécificité du dithizonate de cuivre en faveur de l'argent au pH naturel de l'eau de mer. Nous avons pu constater qu'elle était tout à fait satisfaisante.

Nous avons été amené à travailler sur des volumes d'eau de mer relativement importants de telle façon que la quantité 
d'argent contenue naturellement dans l'échantillon soit suffisante pour être dosée.

c) Méthode adoptée :

Solutions

- Dithizone et chloroforme.

La dithizone est purifiée et le chloroforme distillé, bien que de telles précautions soient en principe inutiles lorsque le réactif utilisé est le dithizonate de cuivre.

Pour purifier la dithizone, nous avons utilisé la méthode de Barnes (1959) : dans un séparateur sont placés $500 \mathrm{~cm}^{3}$ d'eau bidistillée, avec $10 \mathrm{~cm}^{3}$ de $\mathrm{NH}_{4} \mathrm{OH}$ et $50 \mathrm{~cm}^{3}$ de chloroforme. Le tout est agité pendant 30 secondes. L'opération est répétée plusieurs fois en renouvelant le chloroforme jusqu'à ce que la phase organique présente une teinte vert clair. La phase aqueuse est alors acidifiée avec ClH. Ensuite, l'extraction de la dithizone se fait en ajoutant de petites quantités de chloroforme et en les agitant 1 minute environ. Les extraits sont recueillis dans un ballon jaugé de $100 \mathrm{~cm}^{3}$ et complétés au volume avec du chloroforme.

- Dithizonate de cuivre.

La préparation du dithizonate de cuivre est faite en suivant les indications de SANDELL.

Une certaine quantité de la solution de dithizone est placée dans une ampoule à décanter avec une solution de $\mathrm{SO}_{4} \mathrm{H}_{2} 0,05 \mathrm{~N}$, et agitée pendant 1 à 2 minutes. La phase organique est recueillie, puis lavee avec $\mathrm{SO}_{4} \mathrm{H}_{2}$ $0,01 \mathrm{~N}$ pour enlever les gouttelettes de solution aqueuse de cuivre en suspension. Le dithizonate de cuivre est récupéré et on complète au volume avec du chloroforme.

La solution mère de dithizone se conserve bien au froid $\left(+2^{\circ} \mathrm{C}\right)$ et à l'obscurité, à condition que sa surface libre soit couverte d'une couche de chlorhydrate d'hydroxylamine pour éviter l'oxydation.

Il vaut mieux préparer le dithizonate de cuivre juste avant son utilisation. En faisant la solution fille de dithizonate de cuivre, il ne faut pas oublier que la légère miscibilité du chloroforme avec l'eau de mer entraîne une concentration importante de cette solution après l'agitation; celle-ci aura une concentration suffisamment faible (absorption à $550 \mathrm{~m} \mu=0,400$ ), de façon à ce que la précision du spectrophotomètre soit suffisante pour les valeurs trouvées à la nouvelle concentration.

- Solution standard d'argent.

Les solutions d'argent sont fabriquées à partir d'une solution de stockage, obtenue en faisant dissoudre dans $1000 \mathrm{cc}$ 
d'eau distillée sur quartz, 157,4 mgr de $\mathrm{NO}_{3} \mathrm{Ag}$ préalablement desséché à $100^{\circ} .1 \mathrm{cc}$ de la solution mère contient alors $100 \mu \mathrm{gr}$ d'argent.

- Echantillons d'eau de mer.

L'eau de mer est récoltée en surface dans des bouteilles en polyéthylène, à un point déterminé, situé à l'entrée de la rade de Villefranche. Nous avons traité les échantillons dans des délais ne dépassant pas 5 heures.

- Coefficient de concentration dans le chloroforme.

Dans cette série d'expériences, nous n'avons pas pratiqué une saturation préalable de la phase aqueuse. Pour pouvoir comparer directement les valeurs trouvées avec celles obtenues pour la solution de dithizonate de cuivre utilisée comme réactif, il nous a fallu estimer la quantité de chloroforme absorbée par l'eau de mer dans les conditions d'expérience. Nous avons employé la méthode suivante :

$20 \mathrm{~cm}^{3}$ d'une solution de Rouge Soudan III dans le chloroforme, sont agités pendant 5 minutes avec 2 litres d'eau de mer. En comparant la valeur maximum de l'absorption après l'agitation, à celle trouvée avant, il est possible d'obtenir le coefficient de dissolution du chloroforme dans l'eau de mer. Il faut évidemment tenir compte de la substance jaune de KALLE et pratiquer un «blanc». La valeur trouvée a été de 0,51-0,52. Le coefficient par lequel il faut alors multiplier les nouvelles valeurs est 0,515 .

\section{Technique.}

- extraction de l'argent: on verse 2 litres d'eau de mei dans une ampoule à décanter de 3 litres et on leur ajoute $20 \mathrm{cc}$ de dithizonate de cuivre. On agite 5 minutes. L'absorption de la phase organique obtenue est mesurée aux longueurs d'onde de $470 \mathrm{~m} \mu$ et $550 \mathrm{~m} \mu$. Ces valeurs sont rectifiées au moyen d'un blanc de façon à éliminer l'interférence due à la substance jaune de Kalle. Pour rétablir la concentration initiale, il suffit de multiplier ces nouvelles valeurs par le facteur 0,515 . La différence observée entre la valeur à $550 \mathrm{~m} \mu$ ainsi obtenue et celle du témoin à la même longueur d'onde est due à l'argent contenu dans les 2 litres. La teneur en argent est évaluée en comparant cet écart à ceux d'une courbe étalon obtenus dans les mêmes conditions.

- Obtention d'un blanc : un second échantillon de 2 litres d'eau de mer de la même provenance, est agité avec $20 \mathrm{cc}$ de solvant pur, sans réactif, pendant 5 minutes. L'absorption de la phase organique est mesurée aux mêmes longueurs d'onde 
que précédemment et la valeur à $550 \mathrm{~m} \mu$ trouvée est retranchée des valeurs correspondantes des autres échantillons.

- Obtention de la courbe étalon: on ajoute une quantité d'argent connue à 2 nouveaux litres d'eau de mer de la même origine et on pratique une extraction comme précédemment. La valeur de l'absorption obtenue est corrigée puis comparée non pas à celle du réactif mais à celle de la phase organique correspondant aux deux premiers litres d'eau de mer. La différence observée est due à la quantité d'argent ajoutée.

Cette opération est répétée pour des doses variées d'argent, avec chaque fois 2 litres d'eau de mer vierge. Les résultats montrent une diminution de l'absorption à $550 \mathrm{~m} \mu$ proportionnelle à la quantité d'argent ajoutée, donc en accord avec la loi de Beer.

Il arrive qu'il y ait des variations de concentration de la phase organique. Les variations sont toujours très faibles et elles

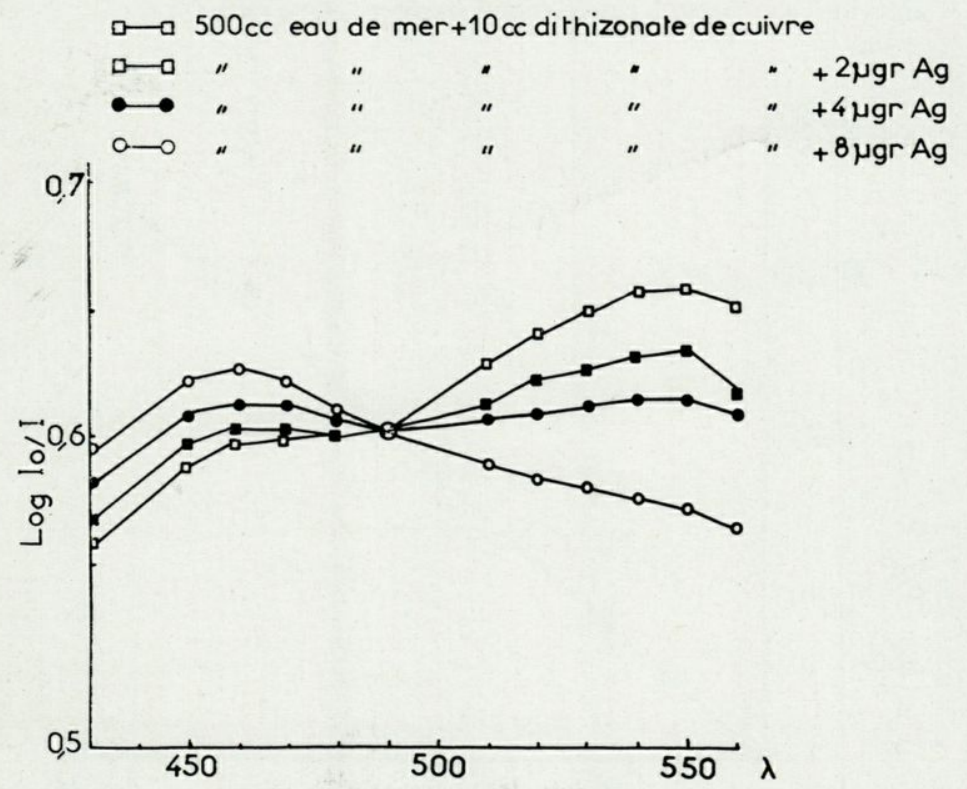

Fig. 6. - Spectres d'absorption obtenus par la méthode des couleurs superposées (réactif : dithizonate de cuivre) lors de l'extraction de 2, 4 et $8 \mu$ gr d'argent ajoutés à $500 \mathrm{~cm}^{3}$ d'eau de mer.

sont sans doute dues aux quelques cc de solution d'argent que nous avons ajouté pour obtenir les valeurs de la courbe étalon. Il est possible d'éliminer l'influence de ces variations. En effet, 
l'examen des différents spectres d'absorption obtenus par cette méthode nous a montré qu'il existait une longueur d'onde $(490 \mathrm{~m} \mu)$ à laquelle la transmittance de la phase organique demeurait remarquablement fixe, quelles que soient les proportions respectives de dithizonate de cuivre et de dithizonate d'argent, à condition que la concentration reste constante (fig. 6). La mesure de la transmittance à cette longueur d'onde permet de déceler les moindres variations de concentration et de les rectifier.

Pour chaque échantillon, nous avons mesuré la transmittance à $490 \mathrm{~m} \mu$, la valeur trouvée étant corrigée comme précédemment. Nous avons fait alors la différence algébrique entre cette valeur et celle du témoin à la même longueur d'onde et nous avons effectué la correction sur les valeurs trouvées aux autres longueurs d'onde.

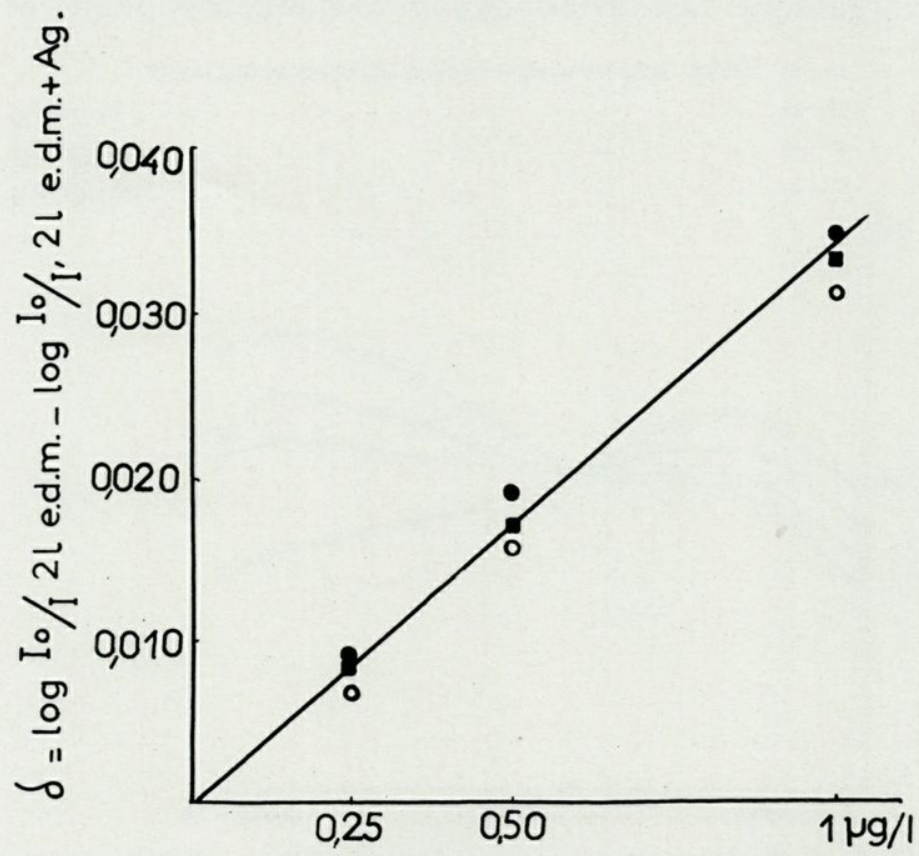

Fig. 7. - Courbe étalon.

Nous n'avons porté sur la figure 7 que les résultats obtenus lors de trois séries de mesures, afin d'avoir une courbe étalon suffisamment claire. En réalité, les valeurs portées sur cette courbe ont été confirmées lors des 10 séries de dosages que nous 
avons réalisées. Dans le tableau suivant, nous indiquons les teneurs en argent des différents échantillons d'eau de mer analysés. Nous pouvons remarquer que lorsque nous avons pratiqué 2 dosages d'une même eau, les résultats ont été très voisins, ce qui est en faveur de notre méthode.

Les teneurs obtenues sont d'ailleurs en rapport avec les données indiquées par Haber, NodDack et NodDack, et Black et MrTCHELL.

\begin{tabular}{c|c|c}
\hline Date & $\begin{array}{c}\text { Nombre } \\
\text { de dosages }\end{array}$ & $\begin{array}{c}\text { Teneur } \\
(\mu \mathrm{gr} / \mathrm{L})\end{array}$ \\
\hline $14 / 11 / 61$ & 1 & 0,12 \\
$15 / 11 / 61$ & 1 & 0,00 \\
$16 / 11 / 61$ & 1 & 0,16 \\
$17 / 11 / 61$ & 2 & $0,10-0,12$ \\
$18 / 11 / 61$ & 1 & 0,25 \\
$20 / 11 / 61$ & 2 & $0,11-0,11$ \\
$21 / 11 / 61$ & 1 & 0,00 \\
$22 / 11 / 61$ & 1 & 0,12 \\
$23 / 11 / 61$ & 1 & 0,00 \\
\hline
\end{tabular}

4. EFFETS BiologiQues DE L'ARGENT

Nous avons procédé avec l'argent comme avec le mercure.

a) Effets sur le développement de Paracentrotus lividus.

Dans notre première série d'essais, la teneur en argent de l'eau de mer nous servant comme référence n'a pas été déterminée.

Lors de l'expérience du 23/11/60, nous avons ajouté à l'eau de mer des quantités d'argent $\left(\mathrm{NO}_{3} \mathrm{Ag}\right)$ variant de 100 à $10 \mu \mathrm{gr}$ par litre. Nous n'avons pas obtenu de plutéus. Une expérience complémentaire $(25 / 11 / 60)$ nous a montré que la segmentation des œufs, très ralentie, se faisait de façon aberrante.

L'addition de $2 \mu \mathrm{gr}$ par litre d'argent, si elle n'empêche pas totalement le développement des œufs, entraîne un retard considérable de ce dernier : sur les 10000 œufs environ que nous avions placés en élevage, une centaine seulement sont arrivés au stade plutéus, et leurs spicules, de taille réduite, étaient difformes. 
Nous avons, au cours des expériences du 5/1/60, du 19/1/60 et du 5/4/60, étudié l'influence de doses d'argent inférieures ou égales à $1 \mu \mathrm{gr}$ par litre. Au cours de ces essais, nous avons mis en évidence un seuil de toxicité et les résultats obtenus ont été confirmés dans les expériences du 19/1/60, 23/3/60 et 20/4/60,

L'addition de $1 \mu$ gr d'argent à l'eau de mer utilisée, provoque une diminution importante de la taille des plutéus, par rapport à celles des témoins. La longueur moyenne des spicules de deux lots témoins est de 39,95 divisions du micromètre $(397 \mu)$ contre 32,64 $(329 \mu)$ pour les deux lots expérimentés. La comparaison statistique des deux moyennes donne pour $t$ une valeur de 21,36 hautement significative $(\mathrm{P}<0,001)$ (fig. $8, \mathrm{~A})$.
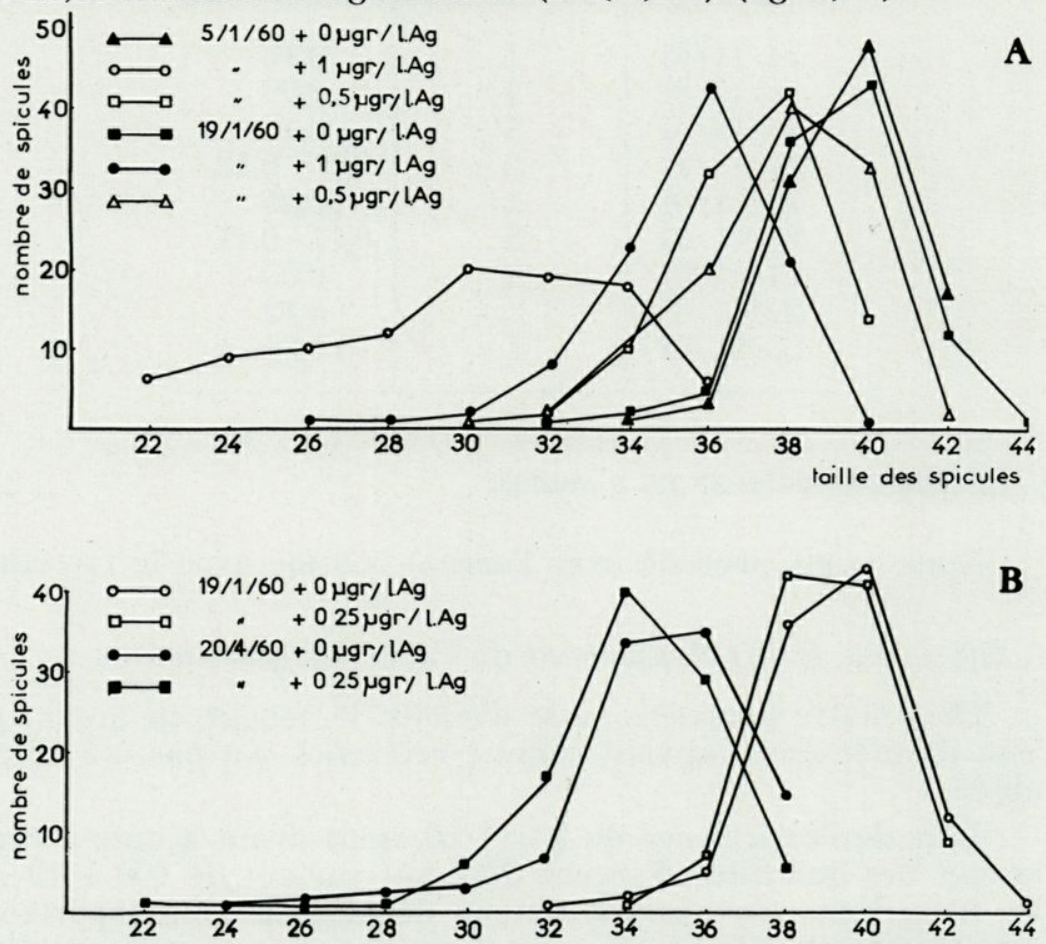

Fig. 8. - Effets de l'argent sur le développement de Paracentrotus lividus.

Avec la même méthode, une quantité de $0,5 \mu$ gr d'argent par litre se montre avoir une action importante. La courbe de fréquence est sensiblement décalée : la longueur moyenne des deux lots expérimentés est de 37,60 divisions du micromètre, soit $379 \mu$, contre $39,35(397 \mu)$ pour les deux lots témoins, $t$ étant alors égal à 9,29 , valeur hautement significative $(\mathrm{P}<0,001)$ (fig. $8, \mathrm{~A})$. 
Comme nous pouvons le voir sur la figure $8, \mathrm{~B}, 0,25 \mu \mathrm{gr}$ par litre d'argent provoquent un très léger décalage de la courbe de fréquence. La comparaison statistique des deux moyennes donne pour $t$ une valeur de 2,19 significative $(\mathrm{P}<0,05)$.

Par contre, l'addition de $0,1 \mu \mathrm{gr}$ par litre ne semble pas avoir entrâné une diminution de la taille des plutéus. Le seuil de toxicité se situe donc à une teneur voisine de $0,25 \mu \mathrm{gr}$ par litre d'argent.

Nous avons repris ces expériences en 1961, avec de l'eau de mer prélevée dans les mêmes conditions mais dont la teneur
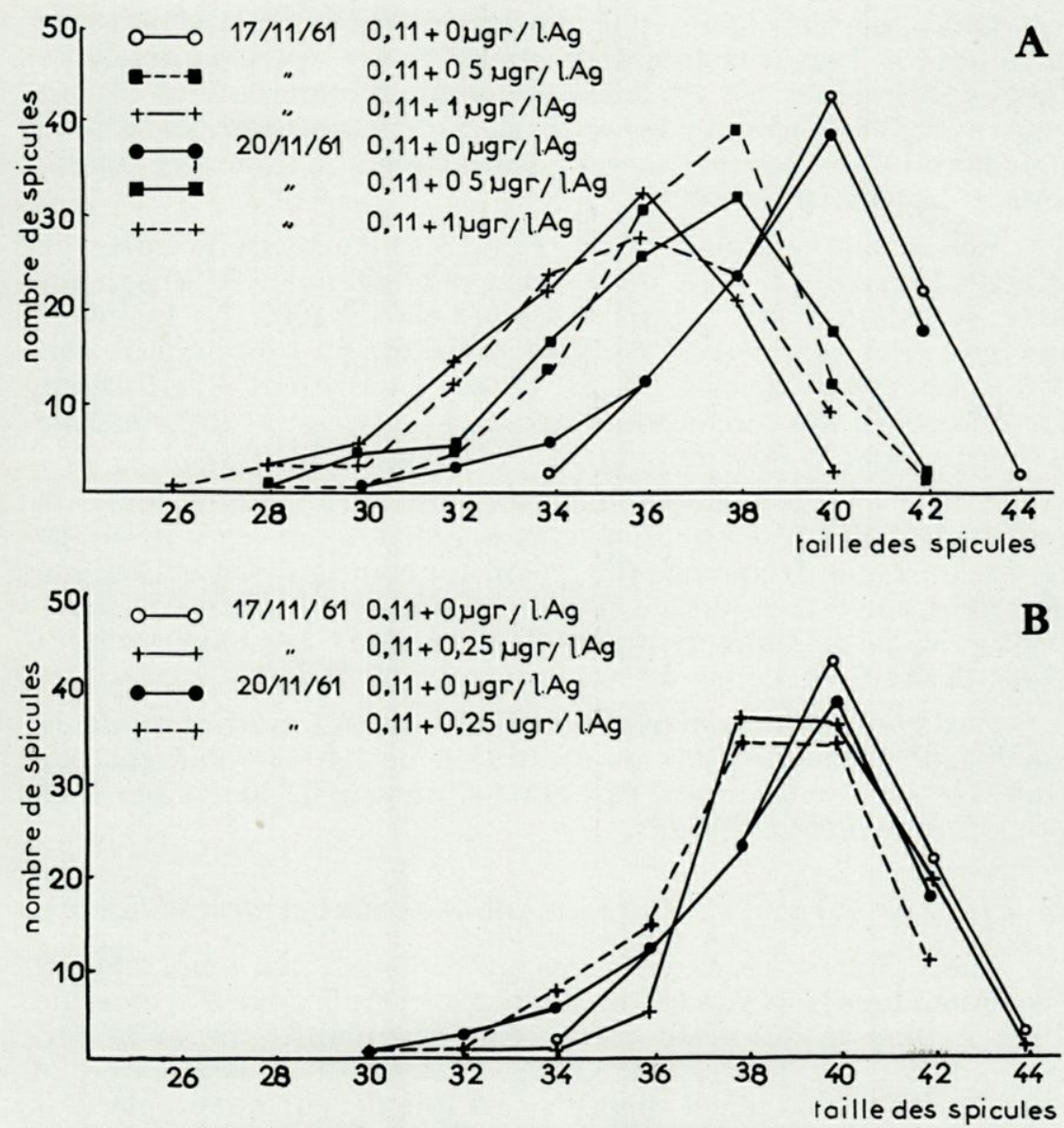

Fig. 9. - Effets de l'argent sur le développement de Paracentrotus lividus (concentration naturelle de l'eau de mer : 0,11 $\mu \mathrm{gr}$ d'Ag/l). 
était déterminée par la réaction au dithizonate de cuivre telle que nous l'avons décrite plus haut. Cette eau était utilisée non filtrée.

Les concentrations en argent des eaux utilisées pour les deux séries d'essais du 17/11/61 et du 20/11/61 étaient identiques : les deux dosages effectués sur chaque prélèvement nous ont donné une teneur en argent de $0,110 \mu \mathrm{gr}$ par litre. Nous avons ajouté à ces eaux des quantités de $1,0,5,0,25$ et $0,1 \mu \mathrm{gr}$ d'argent par litre. Les résultats obtenus sont comparables aux précédents et l'existence du seuil de toxicité vers $0,25 \mu \mathrm{gr}$ par litre est confirmé.

Dans une eau ayant une teneur en argent de 1,11 $\mu \mathrm{gr}$ par litre $(0,11+1 \mu \mathrm{gr})$, la longueur moyenne des spicules des deux lots expérimentés est de 36,06 divisions du micromètre $(313 \mu)$ contre 39,28 $(396 \mu)$ pour les témoins. La comparaison statistique des deux moyennes donne pour $t$ une valeur hautement significative de 16,24 ( $\mathrm{P}<0,001)$.

Les résultats obtenus avec ces mêmes eaux additionnées de $0,5 \mu$ gr d'argent par litre, donc ayant une teneur de $0,610 \mu \mathrm{gr}$ par litre, sont tout à fait comparables à ceux de 1960. La longueur moyenne des spicules est de 36,64 divisions du micromètre, soit $370 \mu$. La valeur de $t, 11,48$, est encore hautement significative. La différence des moyennes correspond environ à un retard de 2 heures dans le développement des pluteus (fig. 9, A).

L'addition de $0,25 \mu \mathrm{gr}$ d'argent par litre, ce qui porte la teneur des eaux à $0,360 \mu$ gr par litre, entraîne un léger décalage de la courbe de fréquence (fig. 9, B). La comparaison statistique des deux moyennes donne pour $t$ une valeur hautement significative de 6,73 , la longueur moyenne des deux lots expérimentés étant de 38,95 divisions du micromtère, soit $393 \mu$.

Par contre, nous n'avons observé aucune variation de la courbe de fréquence lors de l'addition de $0,1 \mu \mathrm{gr}$ d'argent par litre. Le seuil de toxicité serait donc situé vers $0,350 \mu \mathrm{gr}$ par litre pour Paracentrotus lividus.

\section{b) Effets de l'argent sur la fécondation de Paracentrotus lividus.}

Dans toutes les expériences précédentes, les œufs étaient fécondés dans de l'eau de mer naturelle, puis répartis dans de l'eau de mer additionnée d'une certaine quantité de métal. Or, nous avóns vu que certains auteurs avaient constaté que le sperme d'Oursin, placé dans des récipients en argent, perdait rapidement son pouvoir fécondant. Bien que travaillant dans des conditions très différentes de celles de ces auteurs, volume 
d'eau de mer très supérieur et surtout moindre concentration, il nous a paru intéressant de déterminer si l'argent, aux teneurs indiquées, n'était pas capable d'inhiber la fécondation, et si celleci étant possible, l'action de ce métal n'était pas renforcée.

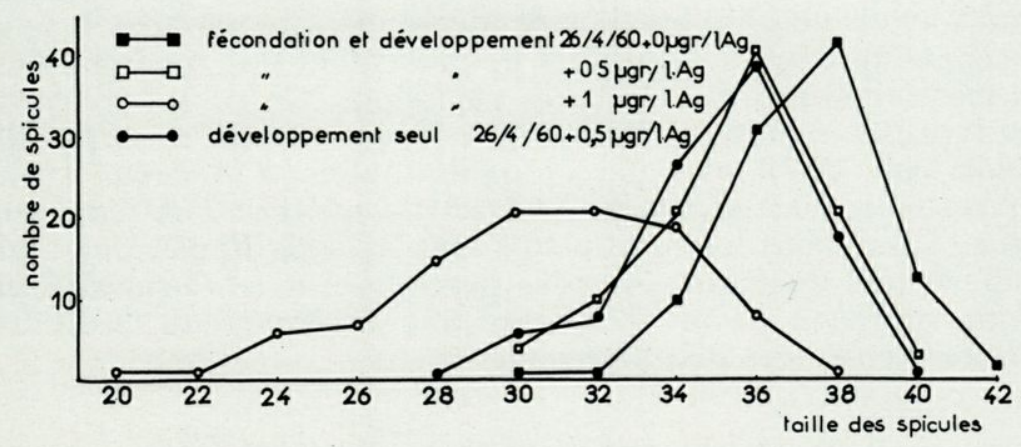

Fig. 10. - Effets de l'argent sur la fécondation de Paracentrotus lividus.

Les deux expériences que nous avons réalisées dans ce sens, nous ont montré qu'il n'en était rien. Ceci est bien visible sur la figure 10. Nous pouvons voir en effet que les œufs de Paracentrotus lividus fécondés dans l'eau de mer additionnée de 1, 0,5 et $0,25 \mu \mathrm{gr}$ d'argent par litre, présentent un retard dans le dévelopement tout à fait comparable à celui des lots de référence fécondés dans l'eau de mer naturelle, mais élevés dans l'eau de mer aux teneurs correspondantes. Des quantités d'argent égales ou inférieures à $1 \mu \mathrm{gr}$ par litre n'empêchent donc pas la fécondation.

c) Permanence des effets de l'argent sur le développement de Paracentrotus lividus.

Il nous a paru également intéressant de déterminer si le retard observé dans la croissance au bout de 40 heures, se maintenait constant au cours du développement. Pour cela, nous avons adopté des temps d'élevage de 40,50 et 60 heures. A la fin de chaque temps, nous avons établi les courbes de croissance d'un lot obtenu dans l'eau de mer additionnée de $1 \mu$ gr d'argent par litre et du lot témoin correspondant (fig. 11). Nous avons pu constater que l'écart entre les deux courbes, observé à 50 heures et 60 heures, était identique à celui existant dès 40 heures. Il semble donc, à la lumière de ces expériences, que le retard soit acquis de manière définitive. Nous n'avons pu augmenter la durée de nos expériences, car au delà de 60 heures, il devient nécessaire d'alimenter les plutéus. 


\section{d) Effets de l'argent sur le développement d'Arbacia lixula.}

Nous savons que la sensibilité aux métaux lourds peut varier d'une espèce à l'autre, à l'intérieur d'un même groupe. Le fait a été démontré pour le cuivre par de nombreux auteurs et nous avons vu qu'il en était de même avec le mercure. Il semble cependant que les Echinides ne présentent pas, ou alors dans une très faible mesure, de telles variations. En ce qui concerne le cuivre, les résultats obtenus par Bovgis avec Paracentrotus lividus, ont été étendus par ce même auteur à Arbacia lixula, et Wilson et Armstrong les ont retrouvés avec Echinus esculentus. Aussi, nous avons effectué avec Arbacia lixula, une série d'expériences analogues à celles pratiquées avec Paracentrotus lividus, de facon à voir s'il existait une différence de sensibilité à l'argent entre ces deux espèces.

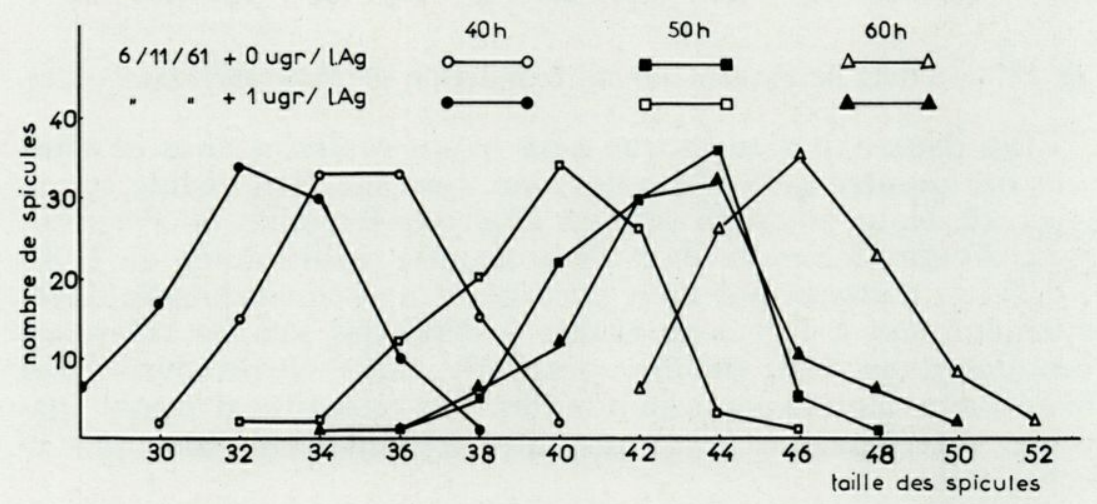

Fig. 11. - Permanence des effets de l'argent sur le développement de Paracentrotus lividus.

La méthode utilisée est la même que précédemment. Comme nous l'avons déjà signalé, nous avons dû allonger le temps d'expérimentation, de façon à obtenir des plutéus de taille suffisante.

Avec de l'eau de mer additionnée de $1 \mu g r$ d'argent par litre, les plutéus de deux lots expérimentés ont une longueur moyenne de 21,30 divisoins du micromètre $(215 \mu)$ contre 23,80 pour les témoins soit $240 \mu$. La comparaison statistique des deux moyennes donne pour $t$ une valeur hautement significative de 11,36 $(\mathrm{P}<0,001)$.

L'addition de 0,75 $\mu \mathrm{gr}$ d'argent par litre entraîne un retard net du développement : les plutéus ont une longueur moyenne (pour les deux lots) de 21,59 divisions du micromètre, soit $218 \mu$, 
et la valeur de $t$ obtenue en la comparant à la moyenne des témoins, s'élève à $9,61(\mathrm{P}<0,001)$.

Comme nous pouvons le constater sur la figure 12, l'addition de $0,5 \mu \mathrm{gr}$ par litre est à peine décelable. La moyenne des deux lots expérimentés est de 23,24 divisions du micromètre $(234 \mu)$, mais la valeur de $t, 2,80$, n'est plus que significative $(\mathrm{P}<0,01)$.

Quant à l'addition de $0,25 \mu \mathrm{gr}$ d'argent par litre, elle n'entraîne aucune perturbation apparente dans le développement et la courbe de fréquence obtenue n'est pas décalée par rapport à celle des témoins.

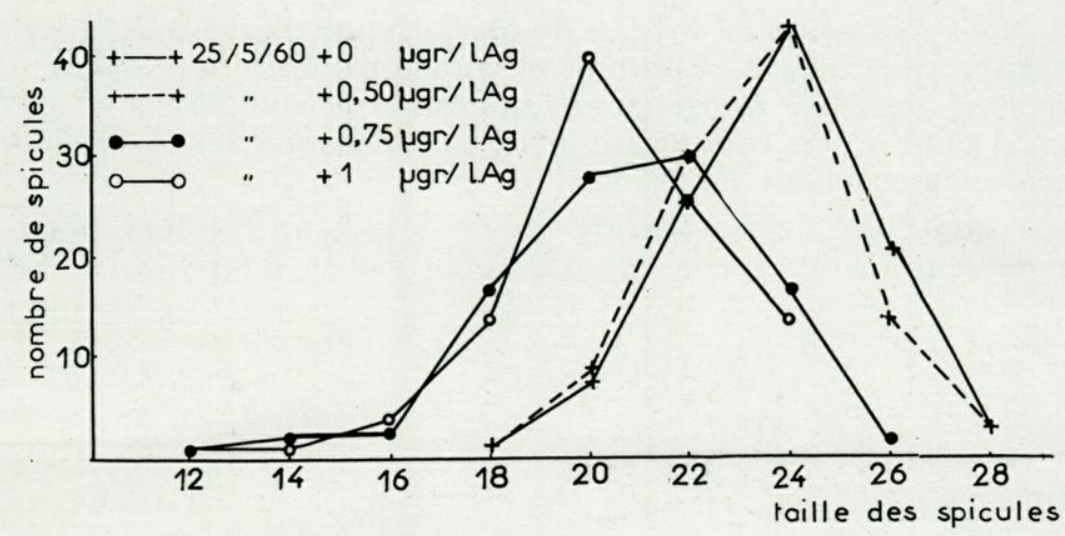

Fig. 12. - Effets de l'argent sur le développement d'Arbacia lixula.

Arbacia lixula aurait donc une résistance à l'argent, supérieure à celle de Paracentrotus lividus, le seuil de toxicité étant alors voisin de $0,5 \mu \mathrm{g}$ par litre.

e) Additivité des actions biologiques de l'argent et du cuivre.

Dans cette partie de notre travail, nous avons étudié l'action conjuguée de l'argent et du cuivre. Nous avons utilisé la même méthode dans nos expériences : à l'eau de mer, nous avons ajouté de faibles quantités de cuivre, d'argent, puis de cuivre et d'argent, et nous avons étudié leur influence sur le développement de Paracentrotus lividus.

Lors de deux séries d'expériences $(23 / 3 / 60$ et $5 / 4 / 60)$, nous avons obtenu les résultats suivants :

Les longueurs moyennes des deux lots expérimentés dans l'eau de mer additionnée de $1 \mu \mathrm{gr}$ d'argent ou de $20 \mu \mathrm{gr}$ de cuivre par litre sont très voisins : respectivement, 30,36 divisions 
$(306 \mu)$ et 30,94 divisions $(311 \mu)$. Les comparaisons statistiques de ces moyennes avec celles des témoins donnent pour $t$ les valeurs hautement significatives de 16,35 et 16,57 .

L'addition de 0,5 ugr d'argent par litre, entraîne une diminution importante de la taille des pluteus dont la moyenne des longueurs s'élève alors à 34,22 divisions, soit $345 \mu$, ce qui donne pour $t$ une valeur de 7,73. Le développement est affecté de façon à peu près identique par l'addition de $10 \mu \mathrm{gr}$ de cuivre par litre. Les plutéus des deux lots expérimentés ont une taille moyenne de 32,88 divisions, soit $332 \mu, t$ étant alors égale à 12,12 , valeur hautement significative $(\mathrm{P}<0,001)$.

Nous avons ajouté à l'eau de mer, 0,5 $\mu$ gr d'argent et $10 \mu \mathrm{gr}$ de cuivre par litre. Les plutéus obtenus ont alors une longueur moyenne de 30,17 divisions du micromètre, soit $304 \mu$, ce qui donne pour $t$, en comparant avec la moyenne des deux lots témoins, une valeur de 19,16.

L'additivité des actions de ces deux métaux est donc pratiquement parfaite et elle apparaît bien sur la figure 13, A. En
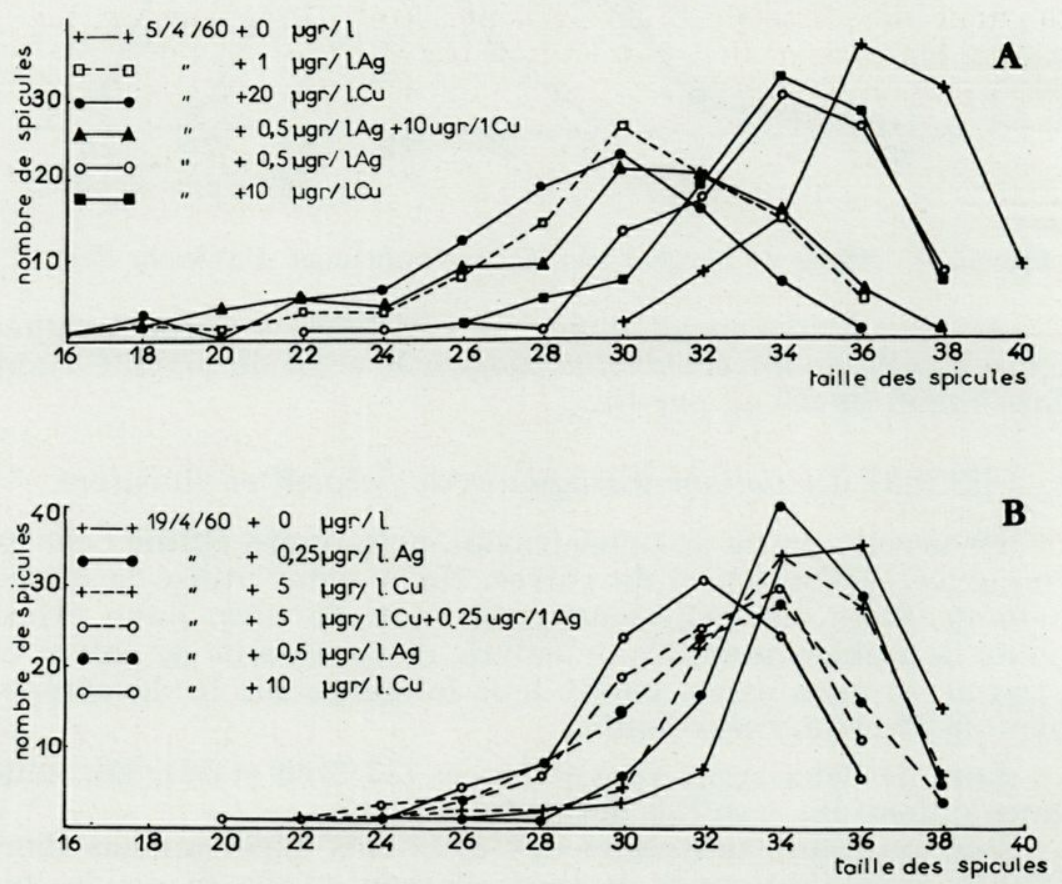

Fig. 13. - Effets d'un mélange argent-cuivre sur le développement de Paracentrotus lividus. 
effet, la différence des moyennes dans ce dernier cas est à peu près identique à celle observée pour $1 \mu \mathrm{gr}$ d'argent ou $20 \mu \mathrm{gr}$ de cuivre par litre. Elle est également proche de la somme des différences des moyennes de la longueur des pluteus obtenus dans de l'eau de mer additionnée séparément de 0,25 بgr d'argent et de $10 \mu \mathrm{gr}$ de cuivre par litre.

Nous avons repris ces expériences avec des quantités plus faibles de cuivre et d'argent $(19 / 4 / 60)$. Nous avons pu également constater, comme cela est évident sur la figure 13, B, que l'additivité était pratiquement parfaite.

En effet, les pluteus obtenus dans de l'eau de mer additionnée de $10 \mu \mathrm{gr}$ de cuivre par litre, ont une taille moyenne de 31,50 divisions du micromètre $(318 \mu)$ contre $32,04(327 \mu)$ pour ceux élevés dans l'eau de mer additionnée de $0,5 \mu$ gr d'argent par litre. Les plutéus obtenus dans la même eau additionnée de $5 \mu \mathrm{gr}$ de cuivre ou de $0,25 \mu \mathrm{gr}$ d'argent par litre, sont également de taille très voisine : $33,98(343 \mu)$ et $34,01(343 \mu)$.

Dans l'eau de mer à laquelle nous avions ajouté $5 \mu \mathrm{gr}$ de cuivre et $0,25 \mu \mathrm{gr}$ d'argent par litre, les plutéus atteignent une longueur moyenne de 31,80 divisions, soit $321 \mu$, comparable à celle obtenue avec $10 \mu \mathrm{gr}$ de cuivre ou $0,5 \mu \mathrm{gr}$ d'argent par litre. La différence observée entre la taille des témoins, $34,62(350 \mu)$ et celle des plutéus de ce dernier lot, est sensiblement égale à la somme des différences des moyennes de la taille des plutéus obtenus séparément avec chaque quantité de métal.

\section{CONCLUSIONS}

$\mathrm{Au}$ cours de notre travail sur l'action biologique de l'argent, nous avons mis au point une méthode d'analyse quantitative de l'argent dans l'eau de mer. Les résultats que nous avons obtenus nous ont montré que la concentration en argent des eaux de surface en Méditerranée devait être très faible $(0,15 \mu \mathrm{gr} / \mathrm{l})$. Nous avons également étudié les effets biologiques de l'argent sur le développement des larves d'Echinides et nous avons mis en évidence :

1) la toxicité de l'argent à des doses faibles, tout à fait comparables aux concentrations observées dans l'eau de mer. Ce métal serait l'un des plus toxiques et, malgré sa présence en très petite quantité, pourrait jouer un rôle important. En effet, en comparant nos résultats avec ceux de Bougis obtenus avec le cuivre et le zinc sur le même matériel, nous avons pu voir que 
l'argent se montrait 80 fois plus actif que le zinc, 20 fois plus que le cuivre et 10 fois plus environ que le mercure.

2) des variations de sensibilité à l'argent chez les Echinides; Arbacia lixula se montre beaucoup plus résistant que Paracentrotus lividus.

3) la possibilité d'une fécondation normale dans des eaux à concentrations en argent toxiques; l'action de l'argent avant la fécondation n'empêche nullement celle-ci de s'effectuer et n'augmente pas les effets du métal.

4) la persistance du retard dans le développement. Il serait cependant intéressant d'étudier l'action de l'argent pendant une durée supérieure à 60 heures de façon à confirmer cette hypothèse.

5) l'additivité parfaite des effets du cuivre et de l'argent. Ce fait est important car il montre qu'il est nécessaire en écologie d'envisager l'ensemble des métaux lourds. Nous avons vu dans notre historique que le mercure amplifiait l'action du cuivre; que l'étain, s'il inhibe l'action de l'argent sur la calcification du plutéus, provoquait d'autres troubles dans le développement. Bougis a montré que les effets du zinc, métal assez peu toxique mais relativement abondant, s'additionnent également à ceux du cuivre, du moins en ce qui concerne les larves d'Oursins. En contrepartie, ces métaux lourds peuvent être masqués dans leurs effets par certains composés chimiques qui les complexent. Ces complexants sont en général des acides aminés. Si la présence de telles substances n'a jamais été démontrée en eau de mer, Peterson, Fred et Domogalla ont reconnu l'existence de la cystéine dans l'eau de lac. Il est donc extrêmement probable que des complexants naturels de cette sorte existent dans l'eau de mer et masquent une fraction plus ou moins importante des métaux lourds et en particulier de l'argent.

Il faut donc faire une remarque importante : si les méthodes d'analyse courantes nous permettent de trouver en quelles proportions sont distribués les métaux lourds dans l'eau de mer, elles ne nous renseignent absolument pas sur la fraction de ces métaux biologiquement active.

Comme nous avons pu le constater lors de l'analyse des travaux d'HaBer et de Lizunov et Lysitsyn, la teneur en argent augmente de façon considérable au voisinage des côtes. Bien que nous manquions de données à ce sujet, il est permis de penser qu'il doit également se produire un enrichissement de l'eau de mer en métaux lourds au contact du sédiment car celui-ci en contient des quantités relativement importantes. Il est donc 
vraisemblable que l'argent joue un rôle important dans l'écologie des peuplements benthiques, côtiers et profonds, séparément ou associés à d'autres métaux lourds. Il serait intéressant d'étudier l'action de ce métal sur la faune interstitielle, qui vit dans des eaux particulièrement riches en métaux toxiques.

Il est également possible que l'argent intervienne dans l'écologie du plankton. Bovgis, dans une publication sur les effets toxiques du cuivre, envisage l'hypothèse d'une explication de la différence entre les eaux «mauvaises 》 et les eaux «favorables 》, constatées par WiLson et ARMstrong, par les teneurs en cuivre de ces eaux. Il met en évidence le remarquable parallélisme qui existe entre les teneurs en cuivre des eaux de la station $\mathrm{E}_{1}$ estimées par Atrins (1953) et la présence, à cette même station, d'eaux «mauvaises » ou «favorables ». Ces eaux étant caractérisées par des populations planctoniques bien différentes, il serait intéressant de vérifier cette hypothèse en déterminant la teneur non seulement en cuivre mais aussi en argent de ces eaux. D'après une publication récente de WiLson et Armstrong (1961), il semble que les métaux lourds et en particulier le cuivre ne soient pas les seuls à intervenir. Rien ne s'oppose cependant au fait que l'argent puisse jouer également un rôle dans l'écologie du plancton vu sa très grande toxicité.

Station Zoologique, Villefranche-sur-Mer

\section{BIBLIOGRAPHIE}

Barnes, M. et Stanbury, F.A., 1948. - The toxic action of copper and mercury salts both separatly and when mixed on the harpacticid copepod, Nitocra spinipes (Boeck). J. Exp. Biol., 25 : 270-75.

Barnes, H., 1959. - Apparatus and Methods of Oceanography I Chemical. G. Allen and Unwin LTD Museum street London.

Black, W.A. et Mitchell, R.L., 1952. - Trace elements in the common brown algae and sea water. J. Marine Biol. Assoc. U. K., 30: 575-84.

Bohn, G. et Drzewina, A., 1928. - Les Convoluta : introduction à l'étude des processus physico-chimiques chez l'être vivant. Ann. Sc. Nat. Zool., 11 : 299-398.

Bohn, G. et Drzewina, A., 1931. - Influence de l'argent métallique sur la calcification des larves d'oursins. C.R. Acad. Sc., 193: 491-93.

Boone, E. et BAAS Becking (L.G.M.), 1931. - Salt effects on eggs and nauplii of Artemia salina. J. Gen. Physiol., 14: 753-763.

Bougis, P., 1959. - Sur l'effet biologique du cuivre en eau de mer. C.R. Acad. Sc., 249 : 326-28.

Bougrs, P., 1961. - Sur l'effet biologique du zinc en eau de mer. C.R. Acad. $S c ., 523$ (4) : 740-741.

Bougrs, P., 1962. - Le cuivre en écologie marine. Coll. Int. "Problemi ecologi delle zone littorali del Mediterraneo», Napoli. 
Chevreuil, 1859. - Présence de l'argent dans l'eau de mer: commentaire après une note de Malaguti. C.R. Acad. Sc., 93.

Clarke, G.L., 1947. - Poisonning and recovery in barnacles and mussels. Biol. Bull., 92 (1) : 73-91.

Clément, H., 1913. - Action de l'argent sur la végétation de l'Aspergillus niger. C.R. Acad. Sc., $74: 749-50$.

Codur, J. et Thiry, G., 1913. - Aspergillus et argent métallique. C.R. Soc. Biol., 74 : 486-87.

Corner, E.D.S. et Rigler F.H., 1958. - The modes of action of toxic agents : Mercuric chloride and $n$ amyl mercuric chloride on crustaceans. J. Marine Biol. Assoc. U.K., 37 (1) : 85-96.

Corner, E.D.S. et Sparrow, B.M.W., 1956. - The mode of action of toxic agents. I : observations on the poisonning of certains crustaceans by copper and mercury. J. Marine Biol. Assoc. U.K., 35 (3) : 531-48.

Corner, E.D.S. et Sparrow, B.M.W., 1956. - The mode of action of toxic agents. II : factors influencing the toxicities of mercury compounds to certain crustacea. J. Marine Biol. Assoc. U.K., 36 : 459-72.

Croghan, P.C., 1958. - The survival of Artemia salina in various media. J. Exp. Biol., 35 (1) : 213-18.

Drzewina, A. et Bohn, G., 1926. - Action de l'argent métallique sur le sperme et les larves d'oursins. C.R. Acad. Sc., 182: 1651-52.

Drzewina, A. et BoHn, G., 1926. - Action antagoniste de l'argent et de l'étain métalliques sur les êtres vivants. C.R. Acad. Sc., 183 : 571-72.

Drzewina, A. et Bohn, G., 1926. - Activation par la lumière des effets de l'argent. C.R. Acad. Sc., 183 : 677-78.

Drzewina, A. et Bohn, G., 1927. - Effets tardifs de l'argent métallique sur les œufs d'oursins. C.R. Soc. Biol., 97: 1522-1524.

Drzewina, A. et Bohn, G., 1929. - Action de l'étain sur les œufs et les larves d'oursins. Arch. Anat. Microsc., 25 : 576-86.

Drzewina, A. et Bohn, G., 1928. - Les Convoluta. Ann. Scien. Nat. Zool., 11 : 299-398.

Fraser, J.H., 1937. - The distribution of Chaetognatha in Scottish water in 1936 with notes on the scottish indicators species. J. Cons. Int. Expl. Mer, 12 : 311-20.

Fraser, J.H., 1952. - The chaetognata and other zooplankton of the Scottish area and their value as biological indicators of hydrographical conditions. Scottish Home dept. Mar. Res., 2 : 1-52.

Galtsoff, P.S., 1932. - The life in the ocean from a biological point of vue. Journ. Wash. Acad. Scien., 22 : 246-257.

Goldberg, E.D., 1960. - Chemists and the oceans. Chymia, 6: 162-179.

GolDSCHMidT, V.M., 1937. - The principles and distribution of chemical elements in minerals and rocks. J. Chem. Soc., 655-73.

Haber, F., 1928. - Das Gold im Meerwasser. Z. Ges. Erdk. Berlin, 3 : 1-12.

HABER, F. et JAENiCKE, J., 1935. - Haber Untersuchungen über das Gold im Meere. Naturwiss., $23: 57$.

HARA J., 1958. - Colorimetric determination of silver by dithizone. Bunseki Kagaku, 7 : 142-47.

Harvey, H.W., 1955. - The chemistry and fertility of sea waters. Cambridge at the University Press.

HitchCoCK, R.D. et STARR, W.L., 1954. - Spectrographic technics as applied to the analysis of sea-water. Appl. Spectroscopy, 8 (1) : 5-17.

HoadLey, L., 1923. - Certains effects of the salts of heavy metals on the fertilisation reaction of Arbacia punctulata. Biol. Bull., 44 : 255-279. 
HoAdley, L., 1930. - Some effects of $\mathrm{HgCl}_{2}$ on fertilized and unfertilized eggs of Arbacia punctulata. Biol. Bull., 58 : 123-144.

Hunter, W.R., 1949. - The poisonning of Marinogrammarus marinus by cupric sulphate and mercuric chloride. J. Exp. Biol., 26, 2 : 113-124.

Hutchinson, G.S., 1957. - A treatise on Limnology. I. Geography, Physics and Chemistry, published by John Wiley and sons Inc. New York.

IRving, H.M.N.H., et ANDREw, G., 1949. - The absorptiometric determination of traces of metals. Reversion : a new procedure. J. Chem. Soc., 537-41.

Irving, H.M.N.H., Cooke, J.J.H., Wardger, S.C., Williams, R.J.P., 1949. Studies with dithizone. Part II : Dithizone as a monobasic acid. J. Chem. Soc., 1847-55.

Inving, H.M.NN.H. and RIDSon, E.J., 1949. — Studies with dithizone. Part I: the determination of traces of Mercury. J. Chem. Soc., 541-47.

Irving, H.M.N.H. and Williams, R.J.P., 1949. - Metal complexes and partition equilibria. J. Chem. Soc., 1841-47.

KorolefF, F., 1950. - Determination of traces of heavy metals in seawater by means of dithizone. Thesis University of Helsingfors.

KRAUSKOPF K.B., 1956. - Factors controlling the concentration of 30 rare metals in sea-water. Geochem. and Cosmochem. Acta, 9: 1-32.

Laroze, A., 1955. - Some aspects of the effect of toxins on fish. An. Fac. Farm. Porto, 15: 77-111.

LILlIE, F.R., 1921. - Studies on fertilisation. X : the effects of copper salts on the fertilization reaction in Arbacia and a comparison of mercury effects. Biol. Bull., 41: 125-143.

LiverSIDGE, A.R., 1895. - On the amount of gold and silver in sea water. J. Proc. Roy. Soc. N.S. Wales, $29: 335-49$ et 350-66.

Lizunov, N.V. et Lisitsyn, A.P., 1955. - Spectrum-analysis data on the composition of the suspension in the Bering Sea. Dorklady Akad. Nauk. S.S.S.R., 104 : 593-96.

Malaguti, 1859. - Présence de l'argent dans l'eau de mer. C.R. Acad. Sc., 49 : $463-64$.

Malaguti, Durocher, 1859. - Observations relatives à la présence de l'argent dans l'eau de mer. C.R. Acad. Sc., 49: 536-37.

Martell, A.E. et Calvin, M., 1956. - Chemistry of Metal Chelate compounds. Prentice Hall Inc.

MunSTER, C.A., 1892. - " On the possibility of extracting precious metals from sea-water ». Norsk. Tck. Tiddsk., 10 : 13-20.

Noddack, I. et Noddack, W., 1940. - Die Haufigkeilen der Schwermetalle im Meerestieren. Ark. Zool., 32 (4) : 1-35.

Pyefinch, K.A. et Мотт, J.C., 1948. - The sensitivity of barnacles and their larvae to copper and mercury. J. Exp. Biol., $25: 276-98$.

RIGHARDS F.A., 1956. - On the state of knowledge of trace elements in the Ocean. Geochem. and Cosmochem. Acta, 10: 241-43.

RichardS, F.A., 1957. - Some currents aspects of chemical Oceanography. Physics and Chemistry of the Earth. Progress Series, 2: 77-128. Pergamon Press London.

Richet, 1881. - De la toxicité comparée des différents métaux. C.R. Acad. Sc., 93 : 649. 
RILEY, G.A., 1943. - The toxicity of heavy metals to fouling organisms. Paper 12, $6^{\mathrm{e}}$ Report from Woods-Hole Oceanographic Institution to the bureau of Ships.

RusSEL, H.W:, 1950. - The poisoning of Marinogammarus marinus by cupric sulfate and mercuric chloride. J. Exp. Biol., 26: 113-24.

Russel, F.S., 1939. - Hydrographical and biological conditions in the North Sea as indicated by plankton organisms. J. Cons. Int. Expl. Mer, 14 : 171-92.

SANDEll, E.B., 1959. - Colorimetric Determination of traces of Metals. Interscience Publishers Inc., New York, 3.

Sauton, B., 1913. - Sur l'action antiseptique de l'or et de l'argent. C.R. Soc. Biol., 74 : 1268-69.

SNEDECOR, G.W., 1956. - Statistical methods applied to experiments in agriculture and biology. Iowa State College Press.

Stock, A. et Cucuel, F., 1934. - Die Verbreitung des Quecksilbers. Naturwiss., 22 : 390-93.

Sverdrup, H.U., Johson, M.W., Fleming, R.H., 1942. - The Oceans; their physics, chemistry and general biology. Prentice Hall.

Waterman, A.S., 1937. - Effect of salts of heavy metals on developpement of the sea urchin Arbacia punctulata. Biol. Bull., 73 (3) : 401-20.

WALNE, P.R., 1956. - Destruction of competative organism on artificial oysters-spat collectors. J. Cons. Int. Expl. Mer, 22: 75-76.

Wichmann, H.J., 1939. - Industr. Engin. Chem., $11: 66$.

WiLson, D.P., 1951. - A biological difference between natural sea waters. J. Marine Biol. Assoc. U.K., 30 : 1-26.

Wilson, D.P. et ARMstrong, F.A.J., 1954. - Biological differences between sea-waters : experiments in 1953. J. Marine Biol. Assoc. U.K., 33 : 347-60.

Wilson, D.P. et ARMStrong, F.A.J., 1952. - Biological differences between sea-waters. J. Marine Biol. Assoc. U.K., 31: 335-49.

Wilson, D.P. et Armstrong, F.A.J., 1958. - Biological differences between sea-waters. J. Marine Biol. Assoc. U.K., 37: 331-48.

WolfF, H., 1954. - Untersuchungen über die Bestimmung von Zink mit Dithizon in biologischen Präparaten. Bioch. Z., 325: 267-79. 